\title{
A relax-and-fix with fix-and-optimize heuristic applied to multi-level lot-sizing problems
}

\author{
Claudio Fabiano Motta Toledo ${ }^{1}$ - Márcio da Silva Arantes ${ }^{1}$. \\ Marcelo Yukio Bressan Hossomi ${ }^{1}$. Paulo Morelato França ${ }^{2}$. \\ Kerem Akartunalı ${ }^{3}$
}

Received: 19 August 2014 / Revised: 6 February 2015 / Accepted: 15 June 2015 /

Published online: 2 July 2015

(C) Springer Science+Business Media New York 2015

\begin{abstract}
In this paper, we propose a simple but efficient heuristic that combines construction and improvement heuristic ideas to solve multi-level lot-sizing problems. A relax-and-fix heuristic is firstly used to build an initial solution, and this is further improved by applying a fix-and-optimize heuristic. We also introduce a novel way to define the mixed-integer subproblems solved by both heuristics. The efficiency of the approach is evaluated solving two different classes of multi-level lot-sizing problems: the multi-level capacitated lot-sizing problem with backlogging and the two-stage glass container production scheduling problem (TGCPSP). We present extensive computational results including four test sets of the Multi-item Lot-Sizing with Backlogging library, and real-world test problems defined for the TGCPSP, where we benchmark against state-of-the-art methods from the recent literature. The computational results
\end{abstract}

$凶$ Claudio Fabiano Motta Toledo

claudio@icmc.usp.br

Márcio da Silva Arantes

marcio@icmc.usp.br

Marcelo Yukio Bressan Hossomi

marcelo.hossomi@usp.br

Paulo Morelato França

paulo.morelato@fct.unesp.br

Kerem Akartunalı

kerem.akartunali@strath.ac.uk

1 Institute of Mathematics and Computer Science, University of São Paulo, Avenida trabalhador são-carlense, 400, Centro, São Carlos, SP 13566-590, Brazil

2 Department of Mathematics and Computer Science, UNESP, R. Roberto Simonsen, 305, CP 266, Presidente Prudente, SP 19060-080, Brazil

3 Department of Management Science, University of Strathclyde,

40 George Street, Glasgow G1 1QE, UK 
show that our combined heuristic approach is very efficient and competitive, outperforming benchmark methods for most of the test problems.

Keywords Lot-sizing $\cdot$ Heuristics $\cdot$ Relax-and-fix $\cdot$ Fix-and-optimize $\cdot$ Backlogging

Mathematics Subject Classification 90C11

\section{Introduction}

Manufacturing systems have been analytically studied for more than a century to achieve better efficiencies and outputs, since manufacturing was a key element, if not "the" key element, of the economic advancement of developed countries. 2013 marks the centenary of the renowned "economic order quantity" formula, which was the first attempt to optimize production quantities under very special conditions. Since then, numerous operations researchers in academia and practice have built many more realistic models and proposed various sophisticated solution methods to tackle lotsizing/production planning problems evident in practice, where decisions such as how much to produce or stock are constrained by various natural limitations such as capacities and setup times.

We investigate two classes of multi-level lot-sizing problems: the multi-level capacitated lot-sizing problem (MLCLSP) with backlogging, and the two-stage glass container production scheduling problem (TGCPSP). The first set of problems, MLCLSP with backlogging, is particularly challenging from a computational point of view, which is also apparent from a number of new lot-sizing problems included in MIPLIB (2010). Moreover, the theoretical question of the full description of the convex hull of the single-item problem with backlogging has remained open for decades until the recent study of Küçükyavuz and Pochet (2009), which indicates the sophistication involved in these problems. Finally, in a practical problem setting, backlogging is never prohibited as all manufacturers will sooner or later fall short of satisfying their customer demands and backlog, and therefore the problem with backlogging presents a more realistic case than the one without. The second set of problems, TGCPSP, represents a real-world short-term production planning and scheduling problem with a first mixed-integer programming (MIP) formulation proposed in Almada-Lobo et al. (2010). The authors in Toledo et al. (2013) improved the previous MIP formulation for the TGCPSP, proposed a hybrid genetic algorithm to solve it and defined sets of complex test problems. TGCPSP does not allow backlogging like MLCLSP, and takes some problem specific characteristics such as production loss costs and sequence-dependent setup times and costs.

The lot-sizing literature can most appropriately be divided into two main areas due to the nature of solution methods used: (i) Exact methods, and (ii) Heuristic methods. Although even the capacitated single-item problem is $\mathcal{N} \mathcal{P}$-hard (see, e.g., Florian et al. 1980) and expectations for optimal solutions diminish as problems become more realistic, exact methods can be very helpful to understand the underlying difficulties in solving these problems. Such methods include valid inequalities (see, e.g., Barany et al. 1984; Miller et al. 2003), extended reformulations (see, e.g., Krarup and Bilde 
1997; Eppen and Martin 1987; Rardin and Wolsey 1993), Lagrangian relaxation (see, e.g., Billington et al. 1986) and Dantzig-Wolfe decomposition (see, e.g., Degraeve and Jans 2007). Most of these studies are mainly focused on simplistic (often single-item) problems, however, many of the developed methods could be extended to realistic problem settings as well. The recent study of Akartunalı and Miller (2012) provides more insight on the complexities apparent in realistic lot-sizing problems, and an extensive discussion of mathematical programming techniques used in the area can be found in Pochet and Wolsey (2006).

Although exact methods are powerful since they provide an exposure of complicating structures and a guarantee on solution quality, they exhibit an important drawback on the computational end: even with the modern fast computers and the state-ofthe-art optimization packages, solving industrial-size lot-sizing problems is a very complicated (and often an impossible) task. To compensate for the computational shortcomings of exact methods and to provide real time solutions to industrial-size problems, heuristic methods have been extensively used in this area, from very simple frameworks to very sophisticated ones, see, e.g., Van Vyve and Pochet (2004), Wu et al. (2011), Kébé et al. (2012), Absi et al. (2013), Toledo et al. (2013), Baki et al. (2014). We also refer the interested reader to Ball (2011) for a recent literature review on general mathematical programming heuristics. Finally, we note that a number of researchers have proposed frameworks using heuristics or meta-heuristics combined with mathematical programming techniques, since the major drawback of heuristic methods is no guarantee of solution quality. Recent results include the ant colony algorithm coupled with reduced MIP solutions for the MLCLSP with overtime proposed by Almeder (2010), the MIP-based and hybrid simulated annealing heuristics for the stochastic lot-sizing problem proposed by Ramezanian and Saidi-Mehrabad (2013), and finally the multi-population genetic algorithm with LP model resolution for the MLCLSP with backlogging proposed by Toledo et al. (2013).

The method described in this paper combines two heuristics based on mathematical programming. Relax-and-fix (RF), a construction heuristic that solves relaxed MIP subproblems sequentially and fixes binary variables throughout the process for speeding it, has been used by a number of researchers for lot-sizing problems: Belvaux and Wolsey (2000) included a basic RF heuristic in their sophisticated lot-sizing solver, whereas Stadtler (2003) proposed a time-oriented RF for MLCLSP with impressive results. More recent applications of RF in the lot-sizing literature include Federgruen et al. (2007) and Akartunalı and Miller (2009), where the former iteratively increase the size of the problem for efficient solutions whereas the latter make use of $(\ell, S)$ inequalities for stronger formulations, outperforming solutions found by Stadtler's heuristic Stadtler (2003). Fix-and-optimize (FO), an improvement heuristic based on MIP, is firstly described in Helber and Sahling (2010) to solve the MLCLSP with lead times and overtime costs. The authors propose product, resource and process-oriented decompositions for the problem, which define subsets of binary variables to be optimized. Seeanner et al. (2013) extend these decomposition ideas to the multi-level lot sizing and scheduling problem, where the neighborhood decomposition search is combined with FO.

We propose a simple and easy-to-implement solution method that also proves to be computationally effective. Contrary to the recent works of Almeder (2010), 
Ramezanian and Saidi-Mehrabad (2013), Toledo et al. (2013) combining complex meta-heuristics with MIP heuristics, our method combines two very simple MIP-based heuristics: RF with fix-and-optimize ("RFFO", as we will refer to in the remainder of the paper). The simplicity is one of the key strengths of the proposed method, allowing any interested researcher or practitioner easily implement it if needed. Moreover, we propose novel ways of building subproblems from the classical rolling time horizon approach, which are important components of RF and FO heuristics, and investigate their effectiveness in practice by extensive computational tests, including over some MIPLIB 2010 instances (MIPLIB 2010).

The method shows impressive computational performance for the majority of difficult test problems of the MCLSP with backlogging, outperforming benchmark methods. Moreover, while many studies such as Helber and Sahling (2010) and Seeanner et al. (2013) explore very specific problem structures for their methodology design, our proposed RFFO framework is designed as generic as possible to avoid taking advantage of a specific problem structure and hence can be extended to other problems if necessary, and in order to support this argument, we have also applied it to TGCPSP, where RFFO was able to find competitive results when compared with the default IBM Ilog Cplex solver and the hybrid genetic algorithm of Toledo et al. (2013).

To summarize, the proposed RFFO method has two main contributions: (i) It is a simple framework combining construction and improvement heuristics, which also returns competitive results in extensive computational tests when compared with state-of-the-art benchmark methods from the recent literature. (ii) The rolling horizon window size is oriented not only by column (i.e., period in lot-sizing, which is the common practice) but also by rows (i.e., families of products) as well as by a combination of columns and rows. Therefore, the method allows rolling windows along with different combinations of columns and rows in the two dimensional matrix representation.

The paper is organized as follows. In the next section, we give a brief mathematical description of the problems under investigation. In Sect. 3, we define in detail our proposed framework, including a discussion of novel ways of building subproblems. Then we present numerical results from extensive computational tests in Sect. 4 with comparisons to two benchmarks methods from recent literature, showing the effectiveness of the proposed methodology. Finally, we conclude with some future directions in Sect. 5.

\section{Multi-level lot sizing problems}

As we discussed earlier, the RFFO approach developed in this paper is not dependent on the problem structure so that it can be adapted to other MIP problems. In this section, we present the MIP formulations of the two classes of multi-level lot-sizing problems. First, we describe the MIP formulation of Toledo et al. (2013), based on the formulation of Akartunalı and Miller (2009), for the MLCLSP with backlogging, and then we describe the MIP formulation for the TGCPSP as presented in Toledo et al. (2013). 


\subsection{MLCLSP with backlogging}

In this paper, we consider MLCLSP with families of products, i.e., multiple products are grouped into families based on their similarities. Since backlogging is a natural practice in manufacturing environments due to capacity limitations, it is allowed for products with external demands.

\section{Parameters}

$J \quad$ Total number of products.

$T \quad$ Total number of periods.

$M \quad$ Total number of machines/resources.

$F \quad$ Total number of families.

$a_{m j} \quad$ Time necessary to produce one unit of product $j$ on machine $m$.

$B_{j t} \quad$ Upper bound for lot-size of product $j$ in period $t$.

$b c_{j} \quad$ Backlogging cost of product $j$.

$C_{m t} \quad$ Total capacity of machine $m$ in period $t$.

$D_{j t} \quad$ Primary demand (external) of product $j$ in period $t$.

$h_{j} \quad$ Holding cost per unit of product $j$ in one period.

$p_{j f} \quad 1$ if product $j$ belongs to family $f$.

$r_{j k} \quad$ Quantity of product $j$ necessary to produce one unit of product $k$.

$s t_{m f} \quad$ Setup time for product family $f$ on machine $m$.

$\delta(j) \quad$ Set of the immediate successors of product $j$.

$\Delta \quad$ Set of the end products.

\section{Variables}

$x_{j t} \quad$ Lot-size of product $j$ in period $t$.

$w_{f t} \quad$ Setup variable of family $f$ in period $t$.

$i_{j t} \quad$ Stock holding quantity of product $j$ in period $t$.

$b_{j t} \quad$ Backlogging quantity of product $j$ in period $t$.

$$
\operatorname{Min} \sum_{j=1}^{J} \sum_{t=1}^{T}\left(b c_{j} \cdot b_{j t}+h_{j} \cdot i_{j t}\right)
$$

Subject to:

$$
\begin{array}{ll}
i_{j t-1}+b_{j t}+x_{j t}=i_{j t}+b_{j t-1}+D_{j t} & \forall j, t \mid j \in \Delta \\
i_{j t-1}+x_{j t}=i_{j t}+\sum_{k \in \delta(j)} r_{j k} \cdot x_{k t} & \forall j, t \mid j \notin \Delta \\
\sum_{j=1}^{J} a_{m j} \cdot x_{j t}+\sum_{f=1}^{F} s t_{m f} \cdot w_{f t} \leq C_{m t} & \forall m, t \\
x_{j t} \leq w_{f t} \cdot B_{j t} & \forall j, f, t \mid p_{j f}=1 \\
x_{j t}, i_{j t}, b_{j t} \geq 0 & w_{f t} \in\{0,1\}
\end{array}
$$

The inventory and backlogging costs are minimized in the objective function (2.1). We note that we do not include setup costs for the sake of simplicity of the model (and 
consider setup times only instead), but the proposed model can be easily modified to include them. The flow balance constraints (2.2) and (2.3) ensure the satisfaction of external and internal demands, respectively, where the external demand for end products can also be satisfied through backlogging. Here, we note that we use these constraints for the sake of simplicity as well as for consistency with the formulations of Akartunalı and Miller (2009), Toledo et al. (2013); however, external demands as well as backlogging can also be included in higher levels of the echelon. The big bucket machine capacities incorporating both variable processing times and fixed setup times in each period are defined by constraints (2.4), where we assume that each product belongs to only one product family and there are product family setup times only (rather than for each product). Constraint (2.5) ensures that a product $j$ cannot be produced (i.e., $x_{j t}=0$ ) if there is no setup for its product family (i.e., $w_{f t}=0$ ). The upper bound for the lot-size of product $j$ in period $t$ is represented by parameter $B_{j t}$, which can be defined using the following definitions of (2.7) and (2.8) (in a similar fashion to Akartunalı and Miller 2009).

$$
\begin{aligned}
B_{j t} & =\min \left(d_{j(1 . . T)}, \frac{C_{m t}-s t_{m f}}{a_{m j}}\right) \\
d_{j(t . . T)} & =\sum_{u=t}^{T} D_{j u}+\sum_{k \in \delta(j)} r_{j k} \cdot d_{k(t . . T)}
\end{aligned}
$$

Note that the Eq. (2.7) bounds the lot-size either by the total demand over the horizon (the first term on the right of the equation) or by the maximum capacity available for production (setup time to be subtracted from the total capacity to identify the production time). Finally, the variable domains are established by constraints (2.6). We note that this formulation can be extended to incorporate other elements of a production system, such as overtime, to make it more realistic. However, we leave it as is for the sake of easier understanding. Finally, we note that due to backlogging allowed to the final period of the horizon, this problem is always feasible. However, as we have observed from our own computational experiences as well as from our discussions with some other researchers, this is a characteristic that makes these problems computationally challenging when attempting to optimize.

\subsection{Two-stage glass container production scheduling problem}

This problem originates from the glass container manufacturing, where a furnace melts the raw material in the first stage of the production process, and molding machines are used in the second stage to finalize the containers. In a typical glass container manufacturer, the daily capacity of the furnace can vary from 100 to 650 ton/day. We refer the interested reader to Toledo et al. (2013) for further technical details of the production process. In short, the TGCPSP is a two-level lot sizing and scheduling problem with parallel machines and sequence-dependent setup costs and times. Different than the problem discussed in the previous section, it does not allow backlogging. 


\section{Parameters}

$C \quad$ : Melting capacity of the furnace in a period (in tonnes).

$\bar{n}_{i k} \quad$ : Maximum number of mold cavities of machine $k$ for product $i$.

$\underline{n}_{i k} \quad$ : Minimum number of mold cavities of machine $k$ for product $i$.

$p_{i k} \quad$ : Amount of product $i$ produced per mold cavity of machine $k$ in a period (in tonnes).

$h_{i} \quad$ : Holding cost for carrying one tonne of product $i$ into the next period.

$c_{i j k} \quad:$ Cost to set up machine $k$ from product $i$ to product $j, i \neq j$.

$s_{i j k} \quad$ : Capacity necessary to set up machine $k$ from product $i$ to product $j, i \neq j$ (in tonnes).

$d_{i t} \quad:$ Demand for product $i$ at the end of period $t$ (in tonnes).

$\omega \quad$ : Penalty cost per tonne of furnace under-utilization.

\section{Decision variables}

$Y_{i t k} \quad: 1$ if product $i$ is assigned to machine $k$ in period $t ; 0$ otherwise.

$Q_{t} \quad: 1$ if the furnace is active in period t; 0 otherwise.

$Z_{i j t k} \quad: 1$ if there is a setup changeover from product $i$ in period $t-1$ to product $j$ in period $t$ on machine $k ; 0$ otherwise.

$N_{i t k} \quad$ : Number of active mold cavities on machine $k$ dedicated to product $i$ in period $t$.

$I_{i t} \quad:$ Inventory of product $i$ at the end of period $t$ (in tonnes).

$\overline{I d}_{t} \quad$ : Idle capacity of the furnace in period $t$ (in tonnes).

$$
\operatorname{Min} \sum_{i, j, t, k} c_{i j k} \cdot Z_{i j t k}+\omega \cdot \sum_{t} \overline{I d}_{t}+\sum_{i, t} h_{i} \cdot I_{i t}
$$

Subject to:

$$
\begin{aligned}
& I_{i t}-I_{i, t-1}+d_{i t}=\sum_{k} p_{i k} \cdot N_{i t k}-\sum_{k, j} s_{j i k} \cdot Z_{j i t k} \quad \forall(i, t) \\
& \sum_{i, k} p_{i k} \cdot N_{i t k}+\overline{I d}_{t}=C \cdot Q_{t} \quad \forall(t) \\
& N_{i t k} \leq \bar{n}_{i k} \cdot Y_{i t k} \quad \forall(i, t, k) \\
& N_{i t k} \geq \underline{n}_{i k} \cdot Y_{i t k} \quad \forall(i, t, k) \\
& \sum_{i} Y_{i t k} \leq 1 \quad \forall(t, k) \\
& Q_{t}=\sum_{i} Y_{i t k} \quad \forall(t, k) \\
& \sum_{i} Y_{i t k} \geq \sum_{i} Y_{i(t+1) k} \quad \forall(t, k) \mid t<T \\
& Y_{j t k}+Y_{i(t-1) k} \leq Z_{i j t k}+1 \quad \forall(i, j, t, k)
\end{aligned}
$$




$$
\begin{aligned}
& \sum_{i, j} Z_{i j t k} \leq Q_{t} \quad \forall(t, k) \\
& p_{i k} \cdot N_{i t k}-\sum_{j} s_{j i k} \cdot Z_{j i t k} \geq 0 \quad \forall(i, t, k) \\
& \left(I_{i t}, \overline{I d}_{t}, Q_{t}\right) \geq 0, N_{i t k} \in \mathbb{Z}_{+},\left(Y_{i t k}, Z_{i j t k}\right) \in\{0,1\}
\end{aligned}
$$

The problem aims to minimize the total cost over the planning horizon that involves inventory and setup costs as well as penalties for the idle capacities of furnaces, as noted in (2.9). The glass container demands have to be fulfilled without backlogging as ensured by (2.10), where the "setup time" (i.e., the number of tonnes of products wasted from the capacity) is also taken account of. The constraint (2.11) enforces the capacity limit of the furnace, and also ensures that the idle time is captured if the furnace is used. The maximum and minimum number of active mold sections in a given machine are enforced by (2.12) and (2.13) respectively, when a product is produced. Each machine can produce at most one product in a time period (2.14), and the two-stage process is synchronized by (2.15), which would activate the furnace if a product is assigned to a machine. If the furnace is deactivated in period $t$, then all associated machines will also be idle in the remainder of the horizon as enforced by (2.16). Constraints (2.17) and (2.18) capture product changeovers and ensure that they can happen only when the furnace is active. Constraint (2.19) enforces that the "setup time" used is not greater than the quantity produced. Finally, (2.20) defines the variable domains.

\section{Proposed heuristic: relax-and-fix with fix-and-optimize}

Here we describe the two heuristics and how they are combined to solve the multilevel lot-sizing problems. For both heuristics, let's consider a matrix $F \times T$ where each entry is a binary variable $w_{f t}$. The RF is a construction heuristic which defines an initial solution by solving several small mixed-integer problems (MIP). This is done by fixing or relaxing most of binary variables, enforcing only few of them to be integer and optimizing them. We call this small set of integer variables as window in the remainder of the paper. The pseudo-code of the RF approach proposed in this paper is summarized in Fig. 1.

The inputs of the RF are the set of binary variables (sol.w), the number of binary variables (windowSize) to be chosen, the selection criteria to choose variables (windowType), the overlap rate of binary variables to be re-optimized (overlap) and the execution time limit (timeLimit). Initially, all binary variables in the RF solution (sol.w) are relaxed which means they can take any value between 0.0 and 1.0. A window is defined as a set that includes a fixed amount (windowSize) of variables (line 2, Fig. 1). The variables inside the window are enforced to be integer in the set $w_{M I P}$ (lines 4 and 10, Fig. 1), while the others are kept relaxed in $w_{L P}$ (lines 5 and 11, Fig. 1). The resulting MIP is then solved (line 7, Fig. 1). Next, a new set of variables (window) is defined, a subset of integer variables is fixed $\left(w_{f i x}\right)$, and another sets of integer and relaxed variables are optimized (lines 8 to 11, Fig. 1). 


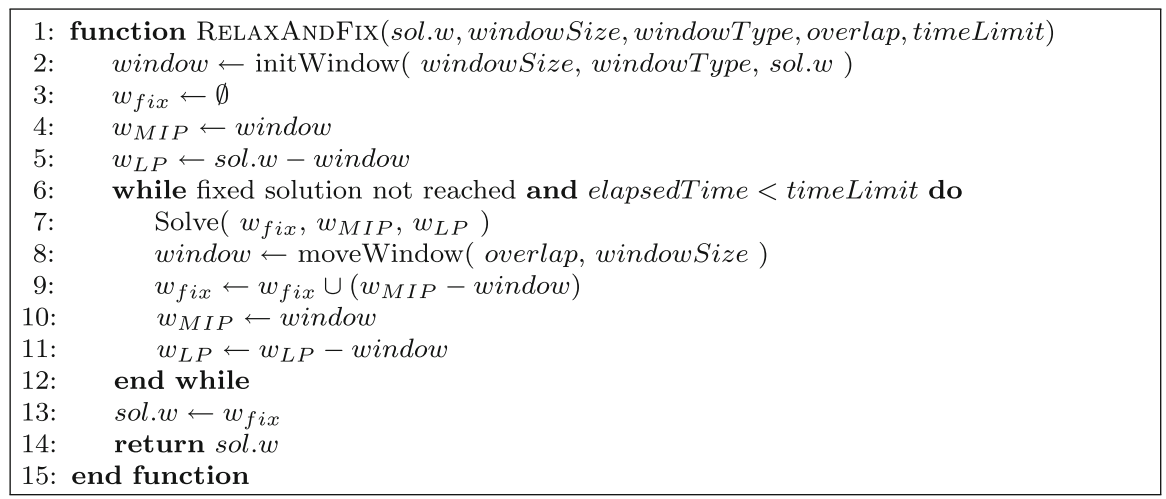

Fig. 1 Pseudocode of relax-and-fix

The window type (windowType) defines how variables are selected to compound the window, as well as how it is moved after each iteration. We propose three different window types: row-wise, in which the window moves along rows; column-wise, in which the window moves along columns; and value-wise, in which the window selects the variables with relaxed values closest to 0.5 . The window moves step variables at each iteration, with step $=\mid$ overlap $*$ window Size $\mid$ (line 8, Fig. 1), where overlap $\in$ $[0,1]$ is the overlap rate defined by the user. Fixing happens to all variables that leave the window in the next iteration (line 9, Fig. 1), and the same number of relaxed variables are enforced to be integer. The algorithm continues processing in this fashion until all variables are fixed. We note that the RF process would benefit if the problem considered, such as the MLCLSP with backlogging, has always a feasible solution; this is a property commonly exploited by other researchers using RF as well [see, e.g., Stadtler (2003) and Akartunalı and Miller (2009)].

Figure 2 shows examples of the three window types as well as how they proceed for windowSize $=5$ and step $=2$. Figure 2 a illustrates the row-wise window, where variables from $w_{F_{1}, T_{1}}$ to $w_{F_{1}, T_{5}}$ are first include in window to be optimized as binary variables. After finding the solution of this MIP, variables $w_{F_{1}, T_{1}}$ and $w_{F_{2}, T_{2}}$ are fixed and leave the windows set, while variables $w_{F_{1}, T_{6}}$ and $w_{F_{2}, T_{1}}$ are enforced to be binary variables. This procedure allows re-optimizing variables $w_{F_{3}, T_{1}}, w_{F_{4}, T_{1}}$ and $w_{F_{5}, T_{1}}$ in this step. A similar idea is applied in the column-wise window as illustrated by Fig. 2 b.

When using the value-wise window, the model is first solved with all variables relaxed so that the relaxed solution is obtained for evaluation. This is shown by the first matrix on the left side of Fig. $2 \mathrm{c}$, where $w_{F_{1}, T_{2}}, w_{F_{4}, T_{2}}, w_{F_{3}, T_{3}}, w_{F_{2}, T_{4}}$ and $w_{F_{3}, T_{4}}$ variables are the closest to 0.5 . Thus, they are selected to be optimized as binary variables in window set. When two or more variables have the same value, those within first columns are preferred. If the variable with same value are in the same column, then first rows will be picked first. In the two-dimensional matrix $F \times T$ defined for lot-sizing problems, this means to choose products in the earlier periods (first columns) and end products (first rows). These criteria are also used to decide how to fix variables, after the MIP problem is solved. For example, variables $w_{F_{1}, T_{2}}$ and $w_{F_{4}, T_{2}}$ are chosen to be fixed (middle matrix, Fig. 2c), once they are in the first column 


\begin{tabular}{|l|cccccc|}
\hline & $\mathrm{T}_{1}$ & $\mathrm{~T}_{2}$ & $\mathrm{~T}_{3}$ & $\mathrm{~T}_{4}$ & $\mathrm{~T}_{5}$ & $\mathrm{~T}_{6}$ \\
\hline $\mathrm{F}_{1}$ & 0.1 & 0.4 & 0.3 & 0.9 & 0.4 & 0.2 \\
$\mathrm{~F}_{2}$ & 0 & 1 & 0.2 & 0.4 & 0.1 & 0.6 \\
$\mathrm{~F}_{3}$ & 1 & 0.2 & 0.5 & 0.4 & 0.6 & 0.3 \\
$\mathrm{~F}_{4}$ & 0.2 & 0.5 & 0.1 & 0.2 & 0 & 0.1 \\
\hline
\end{tabular}

\begin{tabular}{|c|cccccc|}
\hline & $\mathrm{T}_{1}$ & $\mathrm{~T}_{2}$ & $\mathrm{~T}_{3}$ & $\mathrm{~T}_{4}$ & $\mathrm{~T}_{5}$ & $\mathrm{~T}_{6}$ \\
\hline $\mathrm{F}_{1}$ & 1 & 1 & 0 & 1 & 0 & $\mathbf{0 . 6}$ \\
$\mathrm{F}_{2}$ & $\mathbf{0 . 2}$ & 0.9 & 0.2 & 0.3 & 0.1 & 1 \\
$\mathrm{~F}_{3}$ & 0.5 & 1 & 0.2 & 0.8 & 0.9 & 0.4 \\
$\mathrm{~F}_{4}$ & 0 & 0 & 0.3 & 0.7 & 0.3 & 0.2 \\
\hline
\end{tabular}

(a)

\begin{tabular}{|c|cccccc|}
\hline & $\mathrm{T}_{1}$ & $\mathrm{~T}_{2}$ & $\mathrm{~T}_{3}$ & $\mathrm{~T}_{4}$ & $\mathrm{~T}_{5}$ & $\mathrm{~T}_{6}$ \\
\hline $\mathrm{F}_{1}$ & 0.1 & 0.4 & 0.3 & 0.9 & 0.4 & 0.2 \\
$\mathrm{~F}_{2}$ & 0 & 1 & 0.2 & 0.4 & 0.1 & 0.6 \\
$\mathrm{~F}_{3}$ & 1 & 0.2 & 0.5 & 0.4 & 0.6 & 0.3 \\
$\mathrm{~F}_{4}$ & 0.2 & 0.5 & 0.1 & 0.2 & 0 & 0.1 \\
\hline
\end{tabular}

\begin{tabular}{|c|cccccc|}
\hline & $\mathrm{T}_{1}$ & $\mathrm{~T}_{2}$ & $\mathrm{~T}_{3}$ & $\mathrm{~T}_{4}$ & $\mathrm{~T}_{5}$ & $\mathrm{~T}_{6}$ \\
\hline $\mathrm{F}_{1}$ & 1 & 0 & 0.2 & 0.1 & 0.4 & 0.4 \\
$\mathrm{~F}_{2}$ & 1 & 0.9 & 0.2 & 0.3 & 0.1 & 1 \\
$\mathrm{~F}_{3}$ & 1 & 1 & 0.2 & 0.8 & 0.9 & 0.4 \\
$\mathrm{~F}_{4}$ & 1 & 0 & 0.3 & 0.7 & 0.3 & 0.2 \\
\hline
\end{tabular}

(b)

\begin{tabular}{|l|cccccc|}
\hline & $\mathrm{T}_{1}$ & $\mathrm{~T}_{2}$ & $\mathrm{~T}_{3}$ & $\mathrm{~T}_{4}$ & $\mathrm{~T}_{5}$ & $\mathrm{~T}_{6}$ \\
\hline $\mathrm{F}_{1}$ & 0.1 & 0.4 & 0.3 & 0.9 & 0.4 & 0.2 \\
$\mathrm{~F}_{2}$ & 0 & 1 & 0.2 & 0.4 & 0.1 & 0.6 \\
$\mathrm{~F}_{3}$ & 1 & 0.2 & 0.5 & 0.4 & 0.6 & 0.3 \\
$\mathrm{~F}_{4}$ & 0.2 & 0.5 & 0.1 & 0.2 & 0 & 0.1 \\
\hline
\end{tabular}

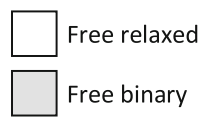

\begin{tabular}{|c|cccccc|}
\hline & $\mathrm{T}_{1}$ & $\mathrm{~T}_{2}$ & $\mathrm{~T}_{3}$ & $\mathrm{~T}_{4}$ & $\mathrm{~T}_{5}$ & $\mathrm{~T}_{6}$ \\
\hline $\mathrm{F}_{1}$ & 1 & 1 & 0 & 0 & 1 & 0 \\
$\mathrm{~F}_{2}$ & 1 & 0.2 & 1 & 0.4 & 0.2 & 0 \\
$\mathrm{~F}_{3}$ & 1 & 0 & 0.9 & 0.7 & 0.5 & 0.2 \\
$\mathrm{~F}_{4}$ & 0.1 & 0 & 0.3 & 0.3 & 0.2 & 1 \\
\hline
\end{tabular}

\begin{tabular}{|c|cccccc|}
\hline & $\mathrm{T}_{1}$ & $\mathrm{~T}_{2}$ & $\mathrm{~T}_{3}$ & $\mathrm{~T}_{4}$ & $\mathrm{~T}_{5}$ & $\mathrm{~T}_{6}$ \\
\hline $\mathrm{F}_{1}$ & 1 & 1 & 0.8 & 0 & 0.1 & 0.8 \\
$\mathrm{~F}_{2}$ & 1 & 0 & 1 & 0.4 & 0.2 & 0 \\
$\mathrm{~F}_{3}$ & 1 & 0 & 0.9 & 0.7 & 0.5 & 0.2 \\
$\mathrm{~F}_{4}$ & 1 & $\mathbf{0 . 2}$ & 0.3 & 0.3 & 0.2 & 1 \\
\hline
\end{tabular}

\begin{tabular}{|c|cccccc|}
\hline & $\mathrm{T}_{1}$ & $\mathrm{~T}_{2}$ & $\mathrm{~T}_{3}$ & $\mathrm{~T}_{4}$ & $\mathrm{~T}_{5}$ & $\mathrm{~T}_{6}$ \\
\hline $\mathrm{F}_{1}$ & 1 & 1 & 0.3 & 0 & 0 & 0.8 \\
$\mathrm{~F}_{2}$ & 0.3 & 0.2 & 0.8 & 0 & 0.2 & 0 \\
$\mathrm{~F}_{3}$ & 1 & 0 & 0 & 1 & 0.5 & 0.2 \\
$\mathrm{~F}_{4}$ & 0.1 & 0 & 0.3 & 0.3 & 0.2 & 1 \\
\hline
\end{tabular}

(c)

\begin{tabular}{|c|cccccc|}
\hline & $\mathrm{T}_{1}$ & $\mathrm{~T}_{2}$ & $\mathrm{~T}_{3}$ & $\mathrm{~T}_{4}$ & $\mathrm{~T}_{5}$ & $\mathrm{~T}_{6}$ \\
\hline $\mathrm{F}_{1}$ & 0.2 & 1 & 0.2 & 0.1 & 0.4 & 0.4 \\
$\mathrm{~F}_{2}$ & 0.8 & 0.9 & 0.2 & 0 & 0.1 & 1 \\
$\mathrm{~F}_{3}$ & 0.5 & 1 & 1 & 1 & 0.9 & 0.4 \\
$\mathrm{~F}_{4}$ & 0 & 0 & 0.3 & 0.7 & 0.3 & 0.2 \\
\hline
\end{tabular}

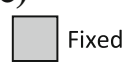

01 Newly included variables

Fig. 2 Relax-and-fix different types of windows: a row-wise; b column-wise; c value-wise. For each window, three iterations are shown in sequence. Window size $=5$, overlap rate $=60 \%$

among variables in the window set. In this step, variables $w_{F_{3}, T_{1}}$ and $w_{F_{1}, T_{5}}$ are now included in window, and $w_{F_{3}, T_{3}}, w_{F_{2}, T_{4}}$ and $w_{F_{3}, T_{4}}$ remain to be re-optimized.

In our framework, the solution built by the RF will be used as the solution to initiate the FO. The steps executed by FO are very similar to RF, where several MIP problems need to be solved. Figure 3 shows the FO pseudo-code. A rolling window, covering windowSize number of variables in $F \times T$ matrix (sol.w), is also defined for FO and these variables are adjusted as binary to be optimized by a solver. However all variables outside the window are kept fixed in the FO heuristic. At each iteration, after solving the MIP subproblem, the window is moved step variables forward with step $=\mid$ overlap $*$ windowSize $\mid$ (line 9, Fig. 3).

FO improves the binary values following row and column directions and hence two window types are defined. The first window type combines the row-wise, which covers the matrix along the rows (Fig. 4a) and column-wise, which does the same along the columns (Fig. 4b) following the same idea defined for RF. However, FO applies both windows types during its execution adjusting windowT ype in lines 2 and 14 (Fig. 3). 


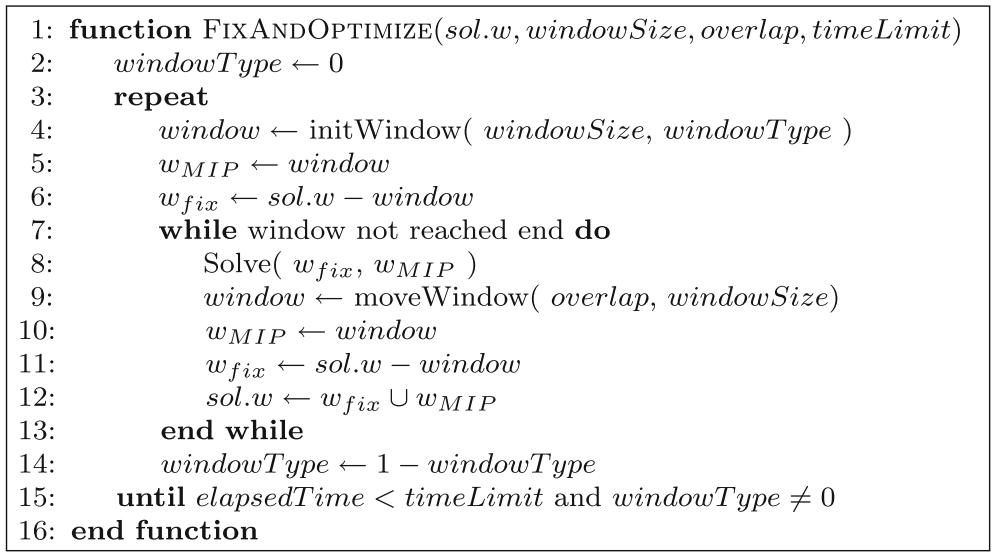

Fig. 3 Pseudocode of fix-and-optimize

\begin{tabular}{|l|l|l|l|l|l|}
\hline 1 & 1 & 0 & 0 & 1 & 0 \\
\hline 1 & 0 & 1 & 0 & 0 & 1 \\
\hline 1 & 1 & 0 & 0 & 0 & 0 \\
\hline 1 & 1 & 1 & 0 & 0 & 0 \\
\hline
\end{tabular}

\begin{tabular}{|l|l|l|l|l|l|}
\hline 0 & 1 & 1 & 0 & 1 & 0 \\
\hline 1 & 1 & 1 & 0 & 0 & 1 \\
\hline 1 & 0 & 1 & 0 & 1 & 1 \\
\hline 1 & 1 & 0 & 0 & 0 & 0 \\
\hline
\end{tabular}

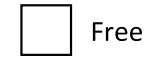

\begin{tabular}{|l|l|l|l|l|l|}
\hline 1 & 1 & 1 & 0 & 0 & 0 \\
\hline 1 & 0 & 1 & 0 & 0 & 1 \\
\hline 1 & 1 & 0 & 0 & 0 & 0 \\
\hline 1 & 1 & 1 & 0 & 0 & 0 \\
\hline
\end{tabular}

\begin{tabular}{|l|l|l|l|l|l|}
\hline 1 & 1 & 0 & 0 & 0 & 0 \\
\hline 1 & 0 & 1 & 0 & 0 & 1 \\
\hline 1 & 1 & 0 & 0 & 0 & 0 \\
\hline 1 & 1 & 1 & 0 & 0 & 0 \\
\hline
\end{tabular}

(a)

\begin{tabular}{|l|l|l|l|l|l|}
\hline 1 & 0 & 1 & 0 & 1 & 0 \\
\hline 1 & 1 & 1 & 0 & 0 & 1 \\
\hline 1 & 0 & 1 & 0 & 1 & 1 \\
\hline 1 & 1 & 0 & 0 & 0 & 0 \\
1
\end{tabular} \mid \begin{tabular}{llllll|l|l|}
1 & 1 & 1 & 0 & 1 & 0 \\
1 & 0 & 1 & 0 & 0 & 1 \\
1 & 1 & 1 & 0 & 1 & 1 \\
1 & 1 & 0 & 0 & 0 & 0 \\
\hline
\end{tabular}

(b)

01 Newly included variables

Fig. 4 Fix-and-optimize: a row-wise and $\mathbf{b}$ column-wise. Three iterations of each direction are shown with window size $=5$ and overlap rate $=60 \%$

In this case, windowType $=0$ means to apply row-wise and windowType $=1$ column-wise.

The second type is a square-wise window that covers the matrix along rows (Fig. 5 a) and columns (Fig. 5b) simultaneously, compounding a square through the matrix. Note that this square overlaps in both sides with the same overlap rate, and step is rounded down to the closest integer multiple of the square side. The square moves along rows and columns during the FO execution according to the windowType value. In this case, windowT ype $=0$ means moving the square in the row direction and window $T$ ype $=1$ moves it in the column direction. 


\begin{tabular}{|l|l|l|l|l|l|}
\hline 0 & 1 & 1 & 0 & 1 & 0 \\
\hline 1 & 1 & 1 & 0 & 0 & 1 \\
\hline 1 & 0 & 1 & 0 & 1 & 1 \\
\hline 1 & 1 & 0 & 0 & 0 & 0 \\
\hline 1 & 1 & 1 & 0 & 0 & 0 \\
\hline 1 & 1 & 1 & 0 & 0 & 1 \\
\hline 1 & 1 & 0 & 1 & 0 & 1 \\
\hline 1 & 0 & 0 & 0 & 0 & 0 \\
\hline
\end{tabular}

\begin{tabular}{|l|l|l|l|l|l|}
\hline 0 & 1 & 1 & 0 & 1 & 0 \\
\hline 1 & 1 & 1 & 0 & 0 & 1 \\
\hline 1 & 0 & 1 & 0 & 1 & 1 \\
\hline 1 & 1 & 0 & 0 & 0 & 0 \\
\hline
\end{tabular}

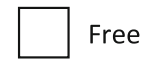

\begin{tabular}{|l|l|l|l|l|l|}
\hline 1 & 1 & 1 & 0 & 0 & 0 \\
\hline 1 & 1 & 0 & 0 & 0 & 1 \\
\hline 1 & 1 & 0 & 0 & 1 & 1 \\
\hline 1 & 0 & 0 & 0 & 0 & 0 \\
\hline 1 & 1 & 1 & 0 & 0 & 0 \\
\hline 1 & 1 & 0 & 1 & 0 & 0 \\
\hline 1 & 1 & 0 & 1 & 1 & 0 \\
\hline 1 & 0 & 0 & 0 & 0 & 0 \\
\hline
\end{tabular}

\begin{tabular}{|l|l|l|l|l|l|}
\hline 1 & 1 & 1 & 0 & 0 & 0 \\
\hline 1 & 1 & 1 & 0 & 0 & 1 \\
\hline 1 & 1 & 0 & 0 & 1 & 1 \\
\hline 1 & 0 & 0 & 0 & 0 & 0 \\
\hline
\end{tabular}

(a)

\begin{tabular}{|l|l|l|l|l|l|}
\hline 1 & 1 & 0 & 0 & 1 & 0 \\
\hline 1 & 1 & 0 & 0 & 0 & 1 \\
\hline 1 & 0 & 1 & 0 & 1 & 1 \\
\hline 1 & 1 & 0 & 0 & 0 & 0 \\
\hline
\end{tabular}

\begin{tabular}{|l|l|l|l|l|l|}
\hline 1 & 1 & 0 & 0 & 1 & 0 \\
\hline 1 & 1 & 0 & 0 & 0 & 1 \\
\hline 1 & 1 & 0 & 0 & 1 & 1 \\
\hline 1 & 0 & 0 & 0 & 0 & 0 \\
\hline
\end{tabular}

(b)

\begin{tabular}{|l|l|l|l|l|l|}
\hline 1 & 1 & 1 & 0 & 0 & 0 \\
\hline 1 & 1 & 1 & 0 & 0 & 0 \\
\hline 1 & 1 & 0 & 1 & 1 & 0 \\
\hline 1 & 0 & 0 & 0 & 0 & 0 \\
\hline
\end{tabular}

01 Newly included variables

Fig. 5 Fix-and-Optimize with square window following: a row and $\mathbf{b}$ column directions. Six iterations of column direction and three of row direction are shown, both with window size $=9$ and overlap rate $=70 \%$

The Relax-and-Fix with Fix-and-Optimize (RFFO) heuristic proposed is summarized in Fig. 6. After the RF execution is complete, FO tries to improve this initial solution until the time limit has been reached. If the improvement achieved by a FO solution did not satisfy a given tolerance $t o l$, the window size is increased by inc variables, a user-defined parameter. Thereafter, the MIP subproblems become larger as an attempt to find better solutions. When using the square window, the increment will affect the window area, making its growth faster when small, and slower when larger. The window area will be rounded to the closest perfect square integer so that the square window can be formed, but the rounded value will not be used in future increment calculations.

\section{Computational results}

The computational tests reported here were run on a PC with Intel i7 processor, 2.6 $\mathrm{GHz}$, and 8GB RAM, and all mathematical models were implemented and solved using IBM ILOG Cplex 12.2 callable library. We have implemented RFFO first to solve the MLCLSP with backlogging, and therefore, we discuss in detail parameter tuning of the method as well as extensive results achieved for the MLCLSP next. Then, 


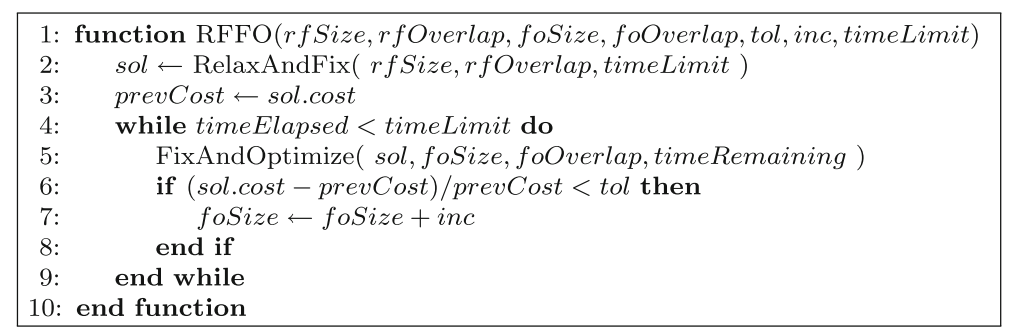

Fig. 6 Pseudocode of RFFO

the application of RFFO to TGCPSP will follow with computational results obtained for instances based on parameters obtained from a glass container manufacturer.

\subsection{Results for MLCLSP with backlogging}

In order to evaluate the effectiveness of our method solving the MLCLSP, we compare the RFFO framework to two state-of-the-art methods from the recent literature: Aheur (Akartunalı and Miller 2009) and LugNP (Wu et al. 2011). The executable codes of these methods were kindly provided by the respective authors so that we could run all methods on the same computer for a fair comparison.

We used all the four test sets (SET1 to SET4) of Multi-LSB (2014) for our computational experiments, where for all problems multi-item and backlogging are allowed. Each of these test sets has 30 instances with 6 machines, 78 products (divided into 11 product families) and 16 periods, except that SET2 instances have 24 periods. A product can be component for only one product in the bill of materials (assembly structure) defined for these instances. The resource utilization factor is 1.05 for SET1 and SET2, 2.0 for SET3 and 1.25 for SET04. This factor determines how much of the total maximum resource capacity is required to supply all the demand, which means a factor greater than 1.0 implies that backlogging in the last period is necessary. The backlogging costs are set to twice the inventory holding cost for SET1 and SET2, and 10 times the inventory holding costs for SET3 and SET4. These characteristics make SET3 and SET4 harder to solve, as also noted by Akartunalı and Miller (2012). Some of the hardest instances from this test are recently included in the MIPLIB 2010 library (MIPLIB 2010) as "open problems". We make a practical remark that very high utilization factors of SET3 and SET4 make these test instances unrealistic in practice. However, the computational challenges they offer as well as the fact that other researchers have used them make these instances appealing, giving us a significant opportunity to benchmark.

All three methods were executed for $100 \mathrm{~s}$ in SET1, $150 \mathrm{~s}$ in SET2 and $300 \mathrm{~s}$ in SET3 and SET4, to remain consistent with the computational times used by Wu et al. (2011) for LugNP, where the Aheur and LugNP results achieved smaller duality gaps against those returned by the branch-and-cut (B\&C) algorithm embedded in Cplex.

Initially we set RFFO parameter values empirically, based on preliminary tests executed over randomly chosen instances, where RF and FO apply value-wise and 
row/column-wise, respectively. RFFO was set with an initial window size of 140 variables for RF windows and 5 for FO windows. Overlap rate for RF was set to $80 \%$, and for FO to $40 \%$. FO improvement tolerance used was set to $5 \%$, and failing to obtain such improvement would increase the window size by 40 variables. Next, the effects of changing these initial parameters were evaluated with computational tests conducted over SET1 to SET4 of MLCLSP as shown by Fig. 7. The new parameter values chosen from the initial ones are indicated with circles, and they allow us to customize RFFO to achieve better results for the benchmark set of instance of MLCLSP. The average deviation for all instances in each set is outlined and such results are compared to LugNP and Aheur. We calculate the deviation (denoted by Dev(\%)) for all instances, using the Eq. (4.1), where Sol ${ }^{\text {Ref }}$ refers to the "Reference" solution, i.e., LugNP and Aheur.

$$
\operatorname{Dev}(\%)=\left(\frac{\operatorname{Sol}^{\text {RFFO }}-\operatorname{Sol}^{\text {Ref }}}{\operatorname{Sol} l^{\text {Ref }}} \cdot 100\right)
$$

The effect of changing parameter values is negligible for the instances of SET1 and SET2, and it also remains limited for the instances of SET3, whereas SET4 instances seem to be in general quite sensitive to parameter changes. A large window for RF seems to be not as efficient as one with 40 variables, where the results for SET4 seem to fluctuate as shown in Fig. 7a, b. The RF overlap rate of $80 \%$ seems to be slightly better than low values (Fig. 7c, d), and increasing the preferable value of $1 \%$ for FO improvement tolerance worsens the results over SET4 (Fig. 7e, f). A large initial FO windows size with 40 variables gives some improvement for all sets (Fig. $7 \mathrm{~g}, \mathrm{~h}$ ), with significant fluctuation for SET4 instances. Once FO fails to improve solutions by $1 \%$, an increment of 10 variables seems to be working best for increasing submodels (Fig. 7i, j). Finally, the overlap rate of $50 \%$ produces slightly better average deviations than the other values (Fig. 7k, 1). We also present the improvement of deviations after each parameter change from the initial settings in Fig. 8, which indicates that these adjustments have the biggest impact on SET4 instances.

As shown on all these cases, the RFFO performs better when RF and FO start solving MIP sub-problems with the RF and FO window size of 40 variables. RF is able to obtain better solutions for FO when $80 \%$ of its variables can be re-optimized (Overlap rate), while FO works better re-optimizing $50 \%$ of its variables. Such behavior seems to be related to the fact that FO is an improvement heuristic and RF is a construction heuristic, so RF needs to review past decision more often to converge to a feasible solution. We also note that we have experimented with the sensitivity of other parameters but seen insignificant differences in many cases. For example, RFFO achieves average values less than $0.1 \%$ different when FO improvement tolerance is set as 5 and $10 \%$ in SET4. An exhaustive finer evaluation of these parameters and experimenting with other test sets might potentially lead RFFO to achieve "optimal" performance. However, as noted earlier, our main focus in this paper is to evaluate strategies regarding choices of decompositions of MIP sub-models apparent in RF and FO, which we will discuss next.

A total of six parameter setups were defined in order to determine which window type combination has better performance for MIP sub-models in RF and FO. Parameter setups \#1 to \#3 use RF row-wise, column-wise and value-wise windows, respectively, 


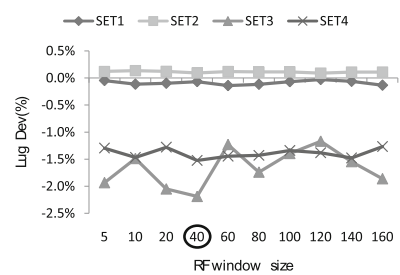

(a)

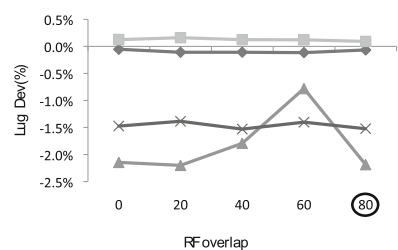

(c)

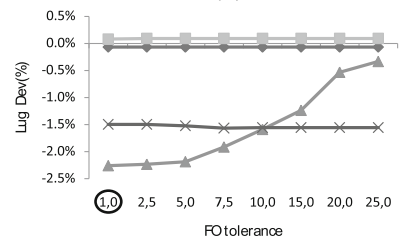

(e)

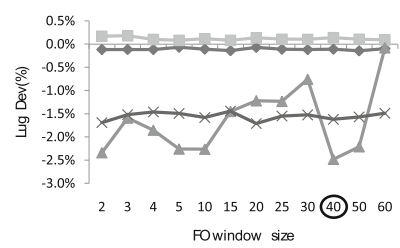

(g)

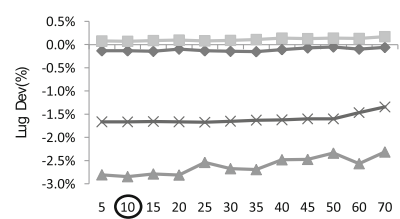

(i)

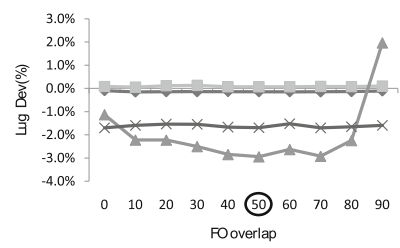

(k)

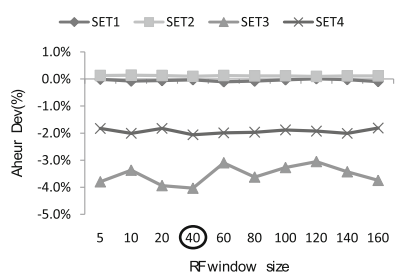

(b)

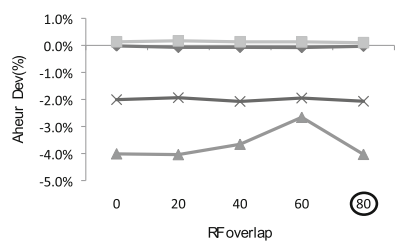

(d)

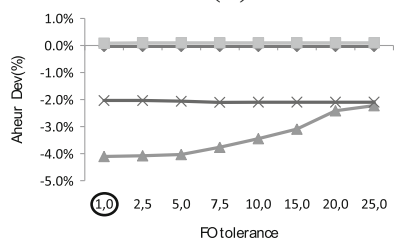

(f)

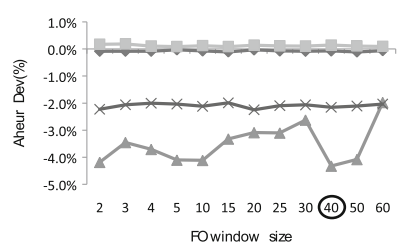

(h)

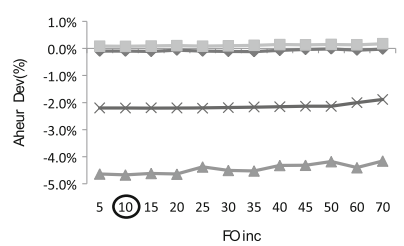

(j)

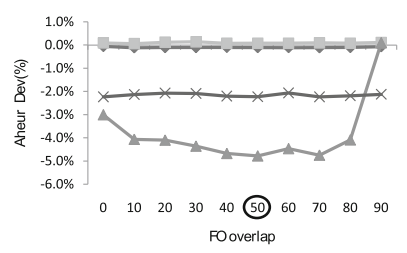

(I)

Fig. 7 Analysis of parameter values: $(\mathbf{a}, \mathbf{b}) \mathrm{RF}$ window size, $(\mathbf{c}, \mathbf{d}) \mathrm{RF}$ overlap rate, $(\mathbf{e}, \mathbf{f}) \mathrm{FO}$ tolerance, $(\mathbf{g}$, h) FO window size, $(\mathbf{i}, \mathbf{j})$ FO increment, $(\mathbf{k}, \mathbf{l})$ FO overlap rate 

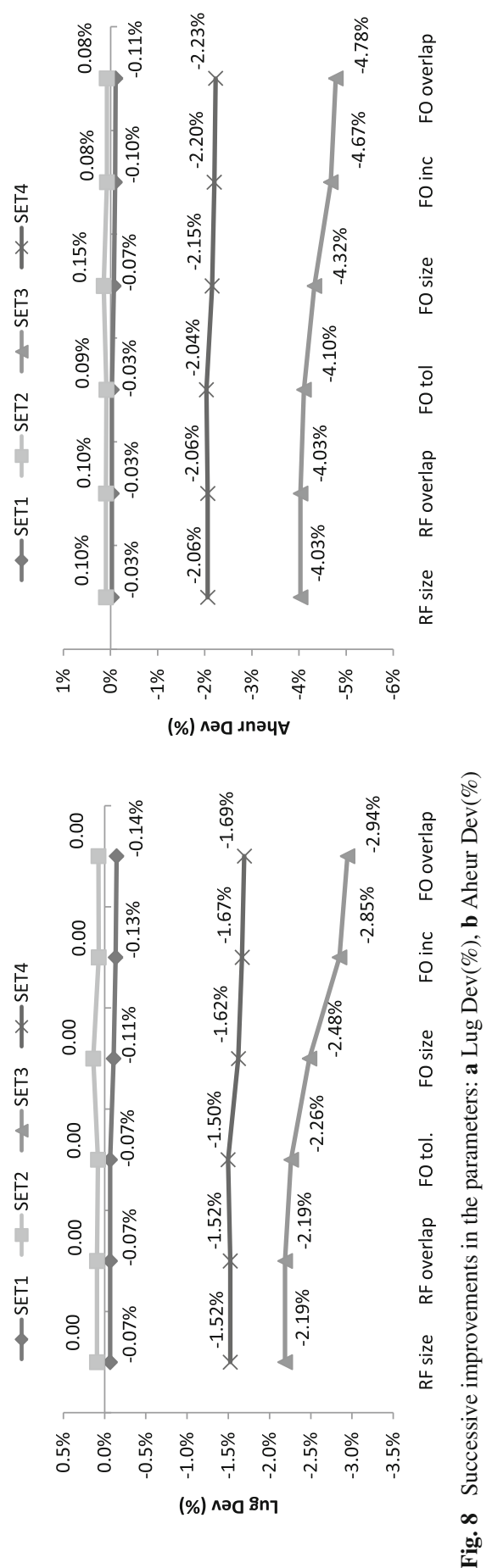
Table 1 Average deviation and number of better solutions in all sets for each window type combination

\begin{tabular}{|c|c|c|c|c|c|c|}
\hline \multirow[t]{2}{*}{ Setup } & \multicolumn{2}{|l|}{ Windows } & \multicolumn{4}{|c|}{ Better solutions } \\
\hline & $\mathrm{RF}$ & $\mathrm{FO}$ & RFFO & Draw & LugNP & Dev. $(\%)$ \\
\hline$\# 1$ & Row & Row/column & 61 & 38 & 21 & -0.49 \\
\hline$\# 2$ & Column & Row/column & 68 & 41 & 11 & -0.98 \\
\hline$\# 3$ & Value & Row/column & 68 & 37 & 15 & -1.18 \\
\hline$\# 4$ & Row & Square & 41 & 36 & 43 & 1.48 \\
\hline$\# 5$ & Column & Square & 57 & 43 & 20 & -0.55 \\
\hline \#6 & Value & Square & 49 & 32 & 39 & 0.20 \\
\hline
\end{tabular}

combined with FO row/column window (first type). Setups \#4 to \#6 use all RF windows combined now with FO square window (second type). We executed all the six setups for all test instances, and the results were compared to LugNP. Table 1 summarizes the results obtained by each setup, showing the number of better solutions found by RFFO, LugNp and the draws. It is considered draw when the deviation of solution values for some instance is less than $0.01 \%$. The last column in Table 1 has the average deviation of RFFO solutions against LugNP for all instances in all sets.

The setup \#3, which combined RF value-wise window and FO row/column-wise window, showed the best performance with an average deviation of $-1.18 \%$ as well as 68 wins over LugNP. The combination of RF row-wise and column-wise with FO row/column-wise also returned improvement from LugNP. However, the FO with square approach seems to be better only when combined with RF column-wise. Thus, for the remainder of computational tests, we used setup \#3.

Next, we present Fig. 9, summarizing how the FO heuristic can improve the initial solution built by RF. It shows the average deviation of the solutions found by RF and RFFO from LugNP and Aheur for SET1 to SET4. RFFO was executed with the parameters values and window types discussed earlier. RF on its own returns on average solutions with less quality compared to the benchmark methods. However, the FO improves these initial solutions significantly for all of these four test sets, in particular for SET3 and SET4.

Finally, we discuss the results comparing the proposed RFFO approach against Aheur and LugNP, as summarized in Table 2. Regarding average percentage improvement, the results for SET1 and SET2 are not necessarily improved by RFFO, where it achieved almost the same performance as the benchmark methods with average deviations around $0.0 \%$. This can be also seen by the high number of draws, but RFFO was able to return better solutions than LugNp and Aheur for both SET1 and SET2. On the other hand, RFFO outperforms the two benchmark methods in SET3 and SET4, achieving more than 4 and $2 \%$ of average improvement, respectively. This is quite significant, since these sets include the most challenging instances. Considering the number of better final solution values, our proposed framework outperformed benchmark approaches for more than 20 out of 30 instances in each of the sets SET3 and SET4. 

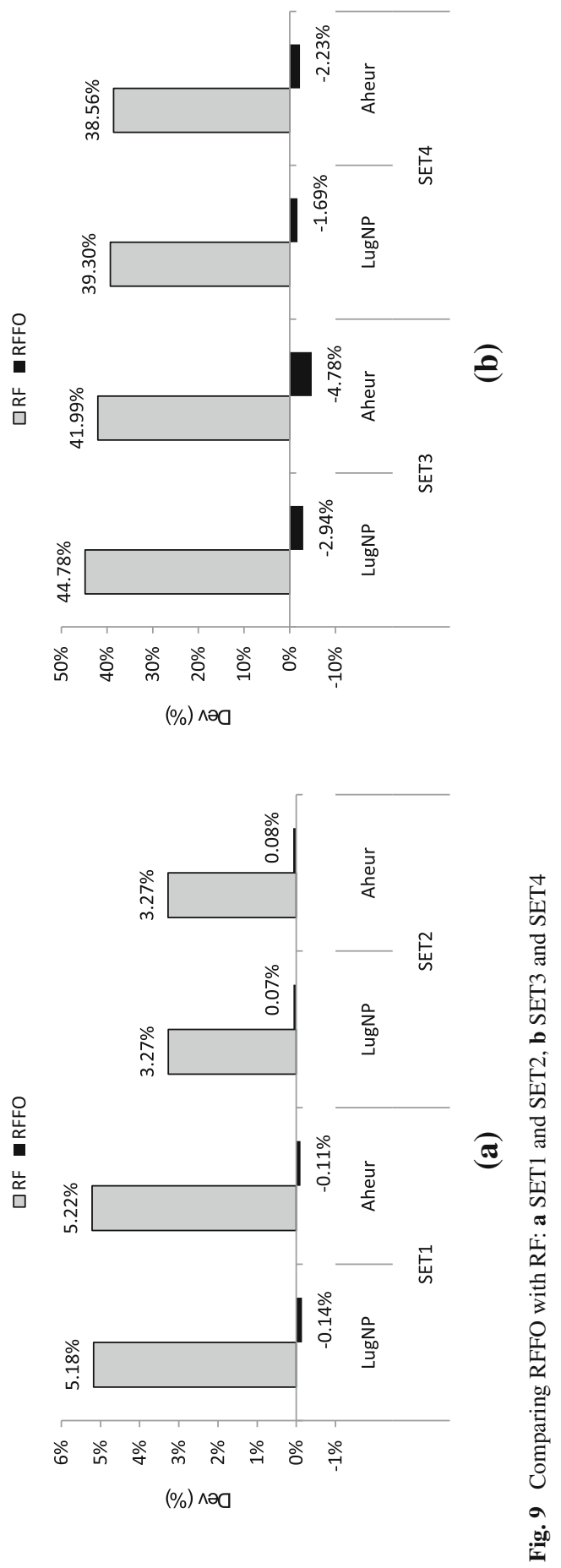
Table 2 Number of better solutions and deviation values by set

\begin{tabular}{|c|c|c|c|c|c|c|c|c|}
\hline \multirow[t]{2}{*}{ Set } & \multicolumn{4}{|c|}{ RFFO versus Aheur } & \multicolumn{4}{|c|}{ RFFO versus LugNP } \\
\hline & RFFO & Draw & Aheur & Dev. $(\%)$ & RFFO & Draw & LugNP & Dev. $(\%)$ \\
\hline SET1 & 8 & 21 & 1 & -0.11 & 10 & 19 & 1 & -0.14 \\
\hline SET2 & 9 & 15 & 6 & 0.08 & 10 & 14 & 6 & 0.07 \\
\hline SET3 & 27 & 0 & 3 & -4.78 & 22 & 0 & 8 & -2.94 \\
\hline SET4 & 28 & 2 & 0 & -2.23 & 26 & 4 & 0 & -1.69 \\
\hline
\end{tabular}

Next we discuss detailed results for each data set, where tables with detailed results for all instances are provided in Appendix. We start with Appendix Tables 4 and 5 showing results for SET1 and SET2 instances, respectively. We also provide the root node lower bounds with $(\ell, S)$ inequalities of Akartunalı and Miller (2012), shown as XLP, to indicate the computational complexity of the instances. It can be noticed that deviations are low and most of them are draws, with several deviations between 1.00 and $0.00 \%$, explaining the low average improvement. Compared to Aheur and LugNP, SET1 has the most positive deviation of $0.24 \%$ and the most negative deviation (best improvement) of $-0.80 \%$ from Aheur and of $-1.83 \%$ from LugNP, respectively. In SET2, it is worth to note that there are more negative than positive values, but the high positive deviations for SET2_23, SET2_1 and SET2_7 are the main reasons for the positive average deviation reported in Table 2. Our computational experience is that the instances in SET1 and SET2 are quite easy to solve in general, and therefore harder to improve, most likely because the results from literature are already very good and close to optimality. This can be verified by the results and comparisons carried on Akartunalı and Miller (2009) and Wu et al. (2011) to support the performance of Aheur and LugNP against B\&C.

Appendix Table 6 shows the results for SET3, where the results dominantly indicate negative deviations, reaching $-11.77 \%$ from Aheur and $-9.18 \%$ from LugNP for the instances SET3_21 and SET3_16, respectively. Another important remark to make is that RFFO improves Aheur and LugNP solutions significantly (more than $5 \%$ ) for 15 and 9 instances, respectively, whereas the worst performance for RFFO is below $3.1 \%$ compared to these two benchmarks (in case of SET3_14, with $1.14 \%$ against Aheur and $3.06 \%$ against LugNP in SET3_29). This is important since SET3 includes hardest to solve instances in these problems.

Appendix Table 7 summarizes the results for SET4. The results are in line with the results of SET3, indicating noticeable negative deviations (though slightly less significant compared to SET3). There is no considerable positive deviation with 2 and 4 results considered draws, respectively, against Aheur and LugNP. Negative deviations reach $-8.53 \%$ against Aheur and $-5.66 \%$ against LugNP, whereas RFFO improves Aheur and LugNP solutions more than $3 \%$ for 8 and 7 instances, respectively.

Finally, we present in Fig. 10 computational performance of different methods (including default Cplex) with extended computational times, where the average value of the best solutions found by each method is given. All methods were executed for 10fold time limits compared to our original time limits, i.e., $1000 \mathrm{~s}$ for SET1 instances 

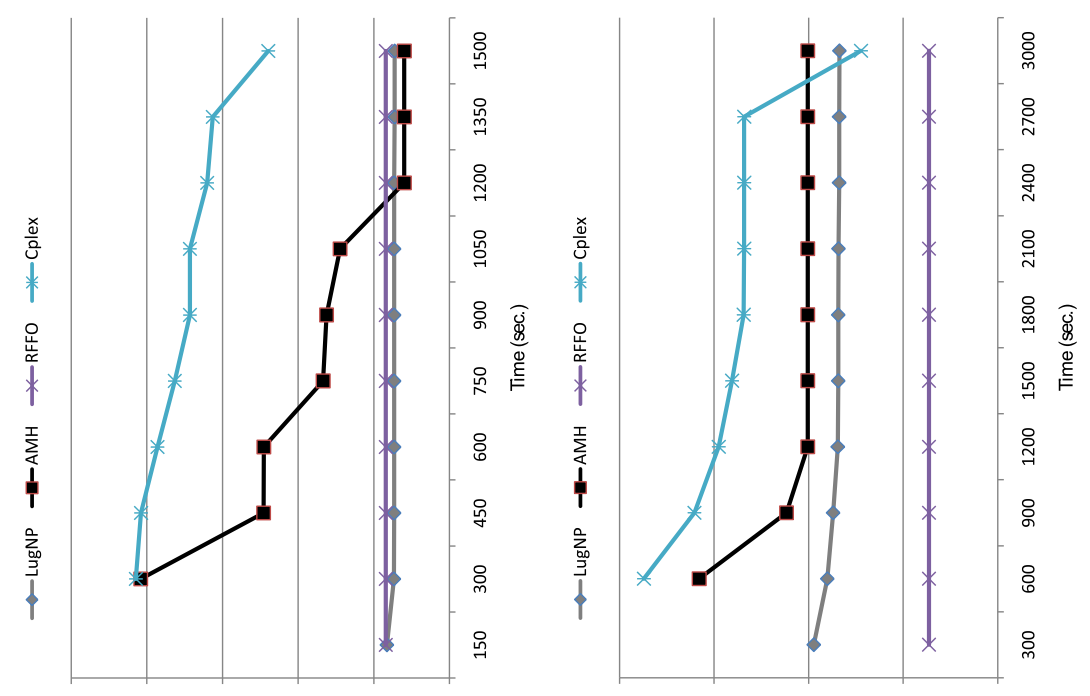

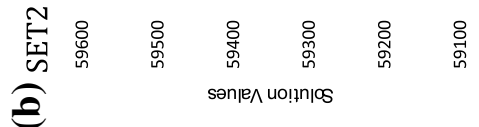

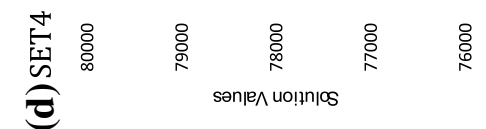
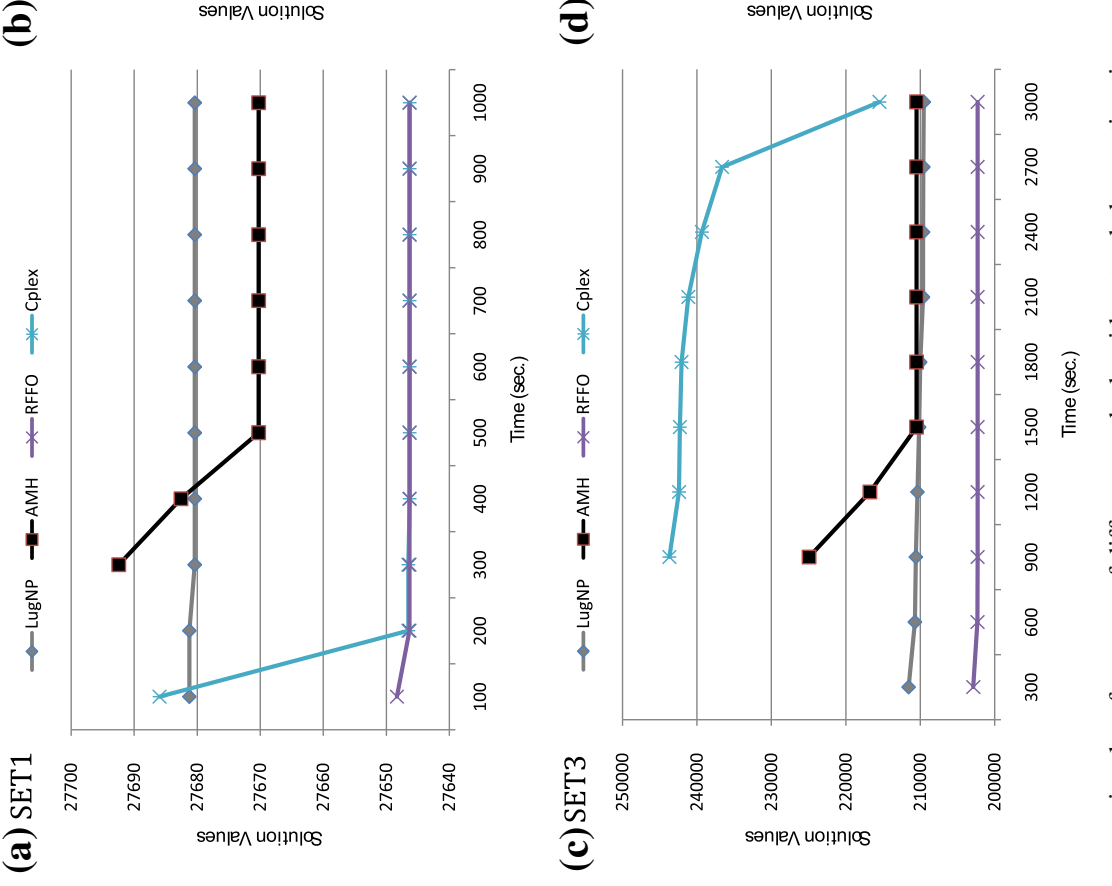

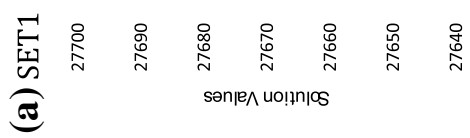

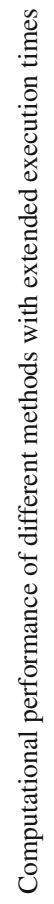

으 
(Fig. 10a), $1500 \mathrm{~s}$ for SET02 instances (10(b)), and $3000 \mathrm{~s}$ for SET03 and SET04 instances (Fig. 10c, d), respectively.

We have omitted some initial values (e.g., Cplex and AMH first values for SET02), as they were out of the scale of the graphs used and would have deteriorated the visualization otherwise. As the graphs indicate, RFFO finds high quality solutions quickly and only improve these solutions slightly during the extended times. Moreover, RFFO solutions over the extended times are only outperformed in SET02, albeit slightly, by LugNP and Aheur, where Aheur is able to do so only after $1200 \mathrm{~s}$. RFFO is always better on average for all other sets showing a more stable performance when compared with the other methods, achieving these solutions very quickly.

\subsection{Results for TGCPSP}

The effectiveness of our method is now evaluated solving the TGCPSP, where one of the key differences compared to previous computational tests is that feasibility of the problems are not guaranteed, which was ensured with the backlogging to the last period in case of MLCLSP. We benchmark our results against those returned by the default Cplex solver and the hybrid genetic algorithm (HGA) of Toledo et al. (2013), which is a custom-designed method specifically for TGCPSP. HGA runs a genetic algorithm (GA) with several populations, where their individuals are hierarchically structured in trees, and integrated with simulated annealing (SA) and the so-called cavity heuristic (CV). SA is applied over the best individual found by the GA at each generation to intensify the search over its neighborhood. CV determines the number of mold cavities and, consequently, the efficiency of the machine during the production process of containers. HGA ran 10 times over each test problem within $1 \mathrm{~h}$, and the same time limit was spent by Cplex to solve each test problem using the model described in Sect. 2.2. More details about the algorithm and parameters used can be found in Toledo et al. (2013).

The test problems, based on data provided by real-world glass container plants, are compounded by 150 artificial and 150 real problems. The artificial set is generated randomly in an academic fashion not necessarily representing a real-world scenario and it involves small to moderate size instances with $T \in\{7,14\}$ days, $K \in\{1,2,3,4,5\}$ machines and $N \in\{5,10,20\}$ products per week. The real set corresponds to actual scenarios that happen in the glass container plants, where the production process involves $T \in\{14,28,56\}$ days with a number of products around 10-90 per week, and $K \in\{2,3,4,5\}$ machines per furnace. For each set of problems, the type of solution returned by the Cplex solver within a $1 \mathrm{~h}$ time limit is used to classify test problems as Optimal (solver returns an optimal solution), Feasible (solver returns a feasible solution without guaranteed optimality), and Unknown (solver does not return a feasible solution). Table 3 indicates some characteristics of these test sets as well as their subsets.

We recall that for the MLCLSP problem discussed in the previous section, RFFO optimized the setup variables $w_{f t}$ combining RF with value-wise window and FO with row/column-wise window, meaning that FO first searches through rows (families $f$ ) and then through columns (periods $t$ ) in the two-dimensional data structure of 
Table 3 TGCPSP problem instances

\begin{tabular}{cccll}
\hline & & & \multicolumn{2}{l}{ Average } \\
\cline { 4 - 4 } Type & Status & \# Instances & CPU(s) & Gap (\%) \\
\hline \multicolumn{2}{l}{ Artificial test problems } & & & \\
O0 & Optimal & 27 & 1.8 & 0.0 \\
O1 & Optimal & 27 & 419 & 0.0 \\
F0 & Feasible & 24 & 3600 & 3.2 \\
F1 & Feasible & 24 & 3600 & 9.8 \\
F2 & Feasible & 25 & 3600 & 17.1 \\
U & Unknown & 23 & 3600 & - \\
Real test problems & & & \\
O0 & Optimal & 3 & 1294 & 0.0 \\
F0 & Feasible & 20 & 3600 & 9.1 \\
F1 & Feasible & 21 & 3600 & 20.6 \\
U & Unknown & 106 & 3600 & - \\
\hline
\end{tabular}

\begin{tabular}{|c|c|c|c|c|c|c|}
\cline { 2 - 7 } \multicolumn{1}{c|}{} & $\mathrm{T}_{1}$ & $\mathrm{~T}_{2}$ & $\mathrm{~T}_{3}$ & $\mathrm{~T}_{4}$ & $\mathrm{~T}_{5}$ & $\mathrm{~T}_{6}$ \\
\hline $\mathrm{I}_{1} \mathrm{~K}_{1}$ & 0 & 0 & 0 & 1 & 1 & 0 \\
\hline $\mathrm{I}_{2} \mathrm{~K}_{1}$ & 1 & 0 & 0 & 0 & 0 & 1 \\
\hline $\mathrm{I}_{3} \mathrm{~K}_{1}$ & 0 & 1 & 1 & 0 & 0 & 0 \\
\hline $\mathrm{I}_{1} \mathrm{~K}_{2}$ & 1 & 1 & 0 & 0 & 0 & 0 \\
\hline $\mathrm{I}_{2} \mathrm{~K}_{2}$ & 0 & 0 & 0 & 0 & 0 & 0 \\
\hline $\mathrm{I}_{3} \mathrm{~K}_{2}$ & 0 & 0 & 1 & 1 & 1 & 1 \\
\hline
\end{tabular}

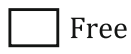

\begin{tabular}{|c|c|c|c|c|c|c|}
\cline { 2 - 7 } \multicolumn{1}{c|}{} & $\mathrm{T}_{1}$ & $\mathrm{~T}_{2}$ & $\mathrm{~T}_{3}$ & $\mathrm{~T}_{4}$ & $\mathrm{~T}_{5}$ & $\mathrm{~T}_{6}$ \\
\hline $\mathrm{K}_{1} \mathrm{I}_{1}$ & 0 & $\mathbf{0}$ & 0 & 1 & 1 & 0 \\
\hline $\mathrm{K}_{2} \mathrm{I}_{1}$ & 1 & 1 & 0 & 0 & 0 & 0 \\
\hline $\mathrm{K}_{1} \mathrm{I}_{2}$ & 1 & 0 & 0 & 0 & 0 & 1 \\
\hline $\mathrm{K}_{2} \mathrm{I}_{2}$ & 0 & 0 & 0 & 0 & 0 & 0 \\
\hline $\mathrm{K}_{1} \mathrm{I}_{3}$ & 0 & 1 & 1 & 0 & 0 & 0 \\
\hline $\mathrm{K}_{2} \mathrm{I}_{3}$ & $\mathbf{0}$ & 0 & 1 & 1 & 1 & 1 \\
\hline
\end{tabular}

(a)

\begin{tabular}{|c|c|c|c|c|c|c|}
\cline { 2 - 7 } \multicolumn{1}{c|}{} & $\mathrm{T}_{1}$ & $\mathrm{~T}_{2}$ & $\mathrm{~T}_{3}$ & $\mathrm{~T}_{4}$ & $\mathrm{~T}_{5}$ & $\mathrm{~T}_{6}$ \\
\hline $\mathrm{I}_{1} \mathrm{~K}_{1}$ & 0 & $\mathbf{0}$ & 0 & 1 & 1 & 0 \\
\hline $\mathrm{I}_{2} \mathrm{~K}_{1}$ & 1 & 0 & 0 & 0 & 0 & 1 \\
\hline $\mathrm{I}_{3} \mathrm{~K}_{1}$ & 0 & 1 & 1 & 0 & 0 & 0 \\
\hline $\mathrm{I}_{1} \mathrm{~K}_{2}$ & 1 & 1 & 0 & 0 & 0 & 0 \\
\hline $\mathrm{I}_{2} \mathrm{~K}_{2}$ & 0 & 0 & 0 & 0 & 0 & 0 \\
\hline $\mathrm{I}_{3} \mathrm{~K}_{2}$ & $\mathbf{0}$ & 0 & 1 & 1 & 1 & 1 \\
\hline
\end{tabular}

(b)

\begin{tabular}{|c|c|c|c|c|c|c|}
\cline { 2 - 7 } \multicolumn{1}{c|}{} & $\mathrm{T}_{1}$ & $\mathrm{~T}_{2}$ & $\mathrm{~T}_{3}$ & $\mathrm{~T}_{4}$ & $\mathrm{~T}_{5}$ & $\mathrm{~T}_{6}$ \\
\hline $\mathrm{K}_{1} \mathrm{I}_{1}$ & 0 & 0 & 0 & 1 & 1 & 0 \\
\hline $\mathrm{K}_{2} \mathrm{I}_{1}$ & 1 & 1 & 0 & 0 & 0 & 0 \\
\hline $\mathrm{K}_{1} \mathrm{I}_{2}$ & 1 & 0 & 0 & 0 & 0 & 1 \\
\hline $\mathrm{K}_{2} \mathrm{I}_{2}$ & 0 & 0 & 0 & 0 & 0 & 0 \\
\hline $\mathrm{K}_{1} \mathrm{I}_{3}$ & 0 & 1 & 1 & 0 & 0 & 0 \\
\hline $\mathrm{K}_{2} \mathrm{I}_{3}$ & 0 & 0 & 1 & 1 & 1 & 1 \\
\hline
\end{tabular}

\begin{tabular}{|c|c|c|c|c|c|c|}
\cline { 2 - 7 } \multicolumn{1}{c|}{} & $\mathrm{T}_{1}$ & $\mathrm{~T}_{2}$ & $\mathrm{~T}_{3}$ & $\mathrm{~T}_{4}$ & $\mathrm{~T}_{5}$ & $\mathrm{~T}_{6}$ \\
\hline $\mathrm{I}_{1} \mathrm{~K}_{1}$ & 0 & 0 & 0 & 1 & 1 & 0 \\
\hline $\mathrm{I}_{2} \mathrm{~K}_{1}$ & 1 & $\mathbf{0}$ & 0 & 0 & 0 & 1 \\
\hline $\mathrm{I}_{3} \mathrm{~K}_{1}$ & 0 & 1 & 1 & 0 & 0 & 0 \\
\hline $\mathrm{I}_{1} \mathrm{~K}_{2}$ & 1 & 1 & 0 & 0 & 0 & 0 \\
\hline $\mathrm{I}_{2} \mathrm{~K}_{2}$ & 0 & 0 & 0 & 0 & 0 & 0 \\
\hline $\mathrm{I}_{3} \mathrm{~K}_{2}$ & 0 & 0 & 1 & 1 & 1 & 1 \\
\hline
\end{tabular}

01 Newly included variables

Fig. 11 Fix-and-optimize: a machine-product-period and b product-machine-period. Three iterations of each direction are shown with window size $=5$ and overlap rate $=60 \%$

$w_{f t}$. Since the setup variables $Y_{i t k}$ of the TGCPSP are three-dimensional, a further elaboration is necessary for the RFFO framework. This does not pose a problem in executing RF with value-wise window, but a strategy to execute FO needs to be adapted from the previous row/column-wise window. Based on our preliminary testing with various options, we concluded to execute FO following first the sequence productmachine-period and then machine-product-period as illustrated by Fig. 11.

In Fig. 11a, the window includes variables selecting indexes by machines $K_{i}$ first followed for items $I_{i}$ (products) and periods $T_{i}$. After to optimize on this way, the window in this three-dimensional data-structure selects variables indexes in Fig. 11b by items followed for machines and periods.

We have executed RFFO for each test instance within the same time limit of $1 \mathrm{~h}$, where we use the initial parameter settings presented in the previous section. First of 
all, to test the flexibility of our approach, we executed RFFO on all the "Unknown" instances that were identified by the default Cplex, which correspond to 15.3 and $70.7 \%$ of the artificial and real test problems, respectively. In the same $1 \mathrm{~h}$ limit, RFFO was able to find solutions for 56.5 and $20.8 \%$ of these unknown instances, respectively, achieving failure rates of 6.67 and $56 \%$ in the overall sets of artificial and real problems, respectively. Although the improvement over Cplex for the unknown artificial instances is significant, the unknown real instances still present a challenge, in particular due to their immense sizes and high number of binary variables (on average 3,329 for real problems). In addition, the involvement of general integer variables complicate these problems significantly and they were not specifically dealt within our RFFO framework in order to preserve the simple structure presented earlier for mixed binary problems. Moreover, the performance might also be affected by the fact that the accessibility of a feasible solution is less straightforward compared to MLCLSP with backlogging, where the simple solution of zero production and backlogging total demand to the last period is always feasible (but costly). We are currently investigating these areas more thoroughly as needed and plan to address these challenges in our future research outcomes.

In order to evaluate the solution quality RFFO can achieve for TGCPSP, we have next executed RFFO for all test instances that are not "Unknown". Using the same time limit of $1 \mathrm{~h}$ as Cplex and HGA, we present our computational results executing RFFO with the initial parameter values $\left(R F F O^{d}\right)$ and with the better parameter setting for the benchmark instances of the MLCLSP $(R F F O)$. Thus, the idea here is to evaluate the performance of RFFO running with the initial values empirically obtained as well as with those parameter values customized to solve MLCLSP instances.

In Fig. 12, we compare all methods for the five subsets of artificial test problems involving 127 instances, where the gap (\%) is calculated by $\operatorname{Gap}(\%)=$ (Upper Bound - LowerBound)/(Upper Bound) using the best solution obtained

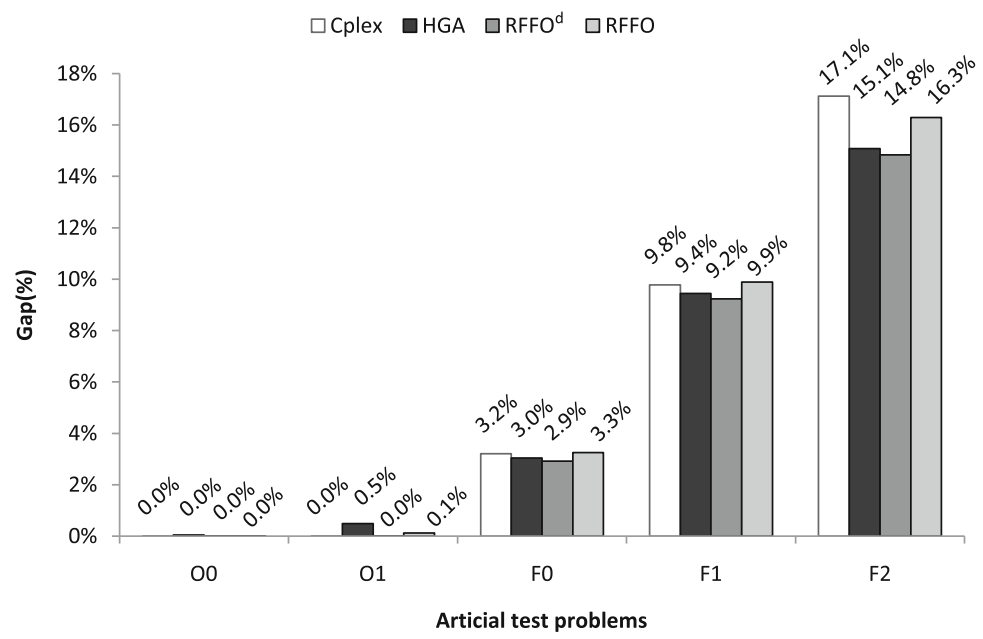

Fig. 12 GAP $(\%)$ comparisons for artificial test problems 


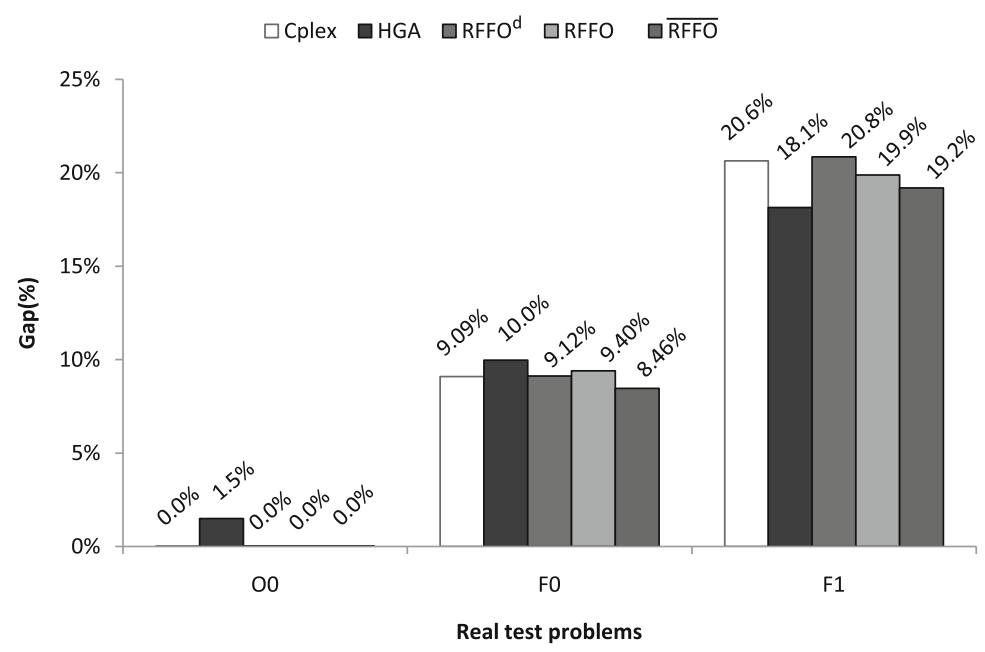

Fig. 13 GAP $(\%)$ comparisons for real test problems

by each method for Upper Bound and the lower bound returned by the branch \& cut algorithm of Cplex for Lower Bound.

As Fig. 12 indicates, $R F F O^{d}$ was able to find optimal solutions in the set of instances, whose optimal values were returned by Cplex ( $O 0$ and $O 1$ ), whereas HGA and $R F F O$ could not return optimal solutions for all test problems belonging to set $O 1$ with average gaps 0.5 and $0.1 \%$, respectively. For the other three subsets of problems, where only feasible solutions were returned by Cplex, all methods had similar performance with regards to solution quality, but $R F F O^{d}$ managed to achieve consistently the lowest average gap value. This is promising, in particular considering that HGA is a custom-designed method for these problems.

In Fig. 13, we present the same evaluation for three subsets of real test problems involving 44 instances. Based on the results obtained by $R F F O^{d}$ for the artificial test problems, we have also implemented a slightly modified version of $R F F O^{d}$, named $\overline{R F F O}$, where the order to optimize variables in the FO is changed. In this case, the setup variables $Y_{i t k}$ are optimized by FO following first the sequence machineproduct-period and then the sequence product-machine-period. Similar to artificial instances, the three RFFO versions were able to find optimal solutions for the real instances, for which Cplex could return their optimal values, while HGA did not manage to find the optimal solution for all instances of this set $O 0$. For the two feasible sets, $R F F O^{d}$ and $R F F O$ return almost the same average gap value as Cplex, whereas the modified version $\overline{R F F O}$ outperforms Cplex and HGA for the set $F 0$ and outperforms Cplex for set $F 1$. On the other hand, HGA generates better solutions on average than RFFO for the set $F 1$, but our results are competitive, considering that HGA is specifically designed for these problems. As the experimentation with $\overline{R F F O}$ indicated, we remark that other changes in RFFO parameters can potentially improve its performance as it was done for the benchmark set of MLCLSP. However, as a general framework, it works effectively. Finally, we note that RFFO is currently 
designed to optimize only binary variables, but a more sophisticated RFFO framework could handle general integer variables of the TGCPSP more efficiently, which we plan to address in near future.

\section{Conclusion}

A hybrid method, RFFO, was proposed by combining two well-known heuristics, RF and FO. A simple combination is proposed, where RF is used to build an initial solution which is further improved by the FO in available computational time. The RFFO is applied to the MLCLSP with backlogging and Two-stage Glass Container Production Scheduling Problem (TGCPSP). Using various test problems available from the literature, the proposed method was benchmarked to state-of-the-art methods from literature: Aheur of Akartunalı and Miller (2009) and LugNP of Wu et al. (2011) for MLCLSP, which are also heuristics based on mathematical programming, and HGA of Toledo et al. (2013) for TGCPSP, which is a genetic algorithm.

In the proposed approach, both heuristics use mathematical programming to solve mixed-integer subproblems defined by a certain amount of binary variables. These variables define a window that moves in the solution matrix using different orientations. Also, the number of binary variables under the window is increased if the solution is not sufficiently improved in a single execution of the FO.

Different strategies to traverse the matrix optimizing the binary variables were proposed and tested, where the best one reported combines a value-wise RF with row/column-wise FO. Thus, the results reported indicate better initial solutions returned by RF when the optimization is focused on relaxed variables closer to 0.5 , followed by FO working better trying to optimize separately rows and columns oriented variables. The best setup found allowed the proposed method significantly outperform the benchmark approaches in two out of four test sets of MLCLSP. However, it was also able to return competitive results in the other two sets. More importantly, the results indicate a better performance of RFFO in the more complex test instances of SET3 and SET4. Similarly, three configuration of RFFO were also able to return competitive results for the more sophisticated problem of TGCPSP, outperforming default Cplex regularly and obtaining better or comparable results with HGA, which is a fast and efficient custom-built method for these problems. We believe that RFFO is overall an effective method for lot-sizing problems with varying characteristics.

As future work, we plan to conduct extensive computational testing on different combinations of parameter values. This would give better insight into sensitivity of different sizes for the MIP's solved by RF and FO, as well as different overlap rate values. We are also currently investigating combining RF and FO with other metaheuristics. For instance, RF could be used to provide different initial solutions if a random criteria is incorporated in the value-wise strategy. Also the proposed FO heuristic could be applied as local search to improve better solutions found by other meta-heuristics. Another area to investigate is the potential improvement of the method if it exploited the specific problem structure. We plan to study this for different settings, e.g., for overtime. 
Finally, we note that the design proposed in this paper is generic and problemindependent. To verify its robustness, we plan to extend this approach to more general MIP problems that naturally have a sequential decision making structure, including problems with general integer variables. In this case, it is in particular our special interest to investigate MIP problems where the RF heuristic could fail to determine an initial solution. Thus, another construction heuristics could be applied taking advantage from the partial solution provided by the proposed RF. We are currently investigating some crew scheduling problems with this framework.

Acknowledgments We would like to thank Dr. Tao Wu for providing us the code of LugNP heuristics used in the comparisons. The work conducted by the first three authors was supported by Fundação de Amparo e Pesquisa do Estado de São Paulo (FAPESP) Projects 2010/10133-0, 2011/15534-5 and 2011/15581-3 and Conselho Nacional de Desenvolvimento Científico e Tecnológico (CNPq) Projects 483474/2013-4 and 312967/2014-4.

\section{Appendix}

See Tables 4, 5, 6, and 7.

Table 4 Comparison for SET1 instances $($ time limit $=100 \mathrm{~s})$

\begin{tabular}{|c|c|c|c|c|c|c|}
\hline \multirow[t]{2}{*}{ SET1 } & \multirow[t]{2}{*}{ XLP } & \multicolumn{3}{|c|}{ Solution values } & \multicolumn{2}{|c|}{ Deviation (\%) } \\
\hline & & Aheur & LugNP & RFFO & Aheur & LugNP \\
\hline 1 & 17,888 & $22,382.5$ & $22,460.7$ & $22,382.1$ & 0 & -0.35 \\
\hline 2 & 23,534 & $27,584.8$ & $27,584.8$ & $27,584.6$ & 0 & 0 \\
\hline 3 & 21,227 & $25,187.3$ & $25,187.3$ & $25,246.6$ & 0.24 & 0.24 \\
\hline 4 & 22,232 & $26,334.7$ & $26,334.7$ & $26,334.7$ & 0 & 0 \\
\hline 5 & 21,446 & $25,145.5$ & $25,145.5$ & $25,145.8$ & 0 & 0 \\
\hline 6 & 22,974 & $26,667.4$ & $26,770.8$ & $26,667.5$ & 0 & -0.39 \\
\hline 7 & 20,360 & $24,123.8$ & $24,123.8$ & $24,124.2$ & 0 & 0 \\
\hline 8 & 25,582 & $29,640.4$ & $29,640.4$ & $29,639.8$ & 0 & 0 \\
\hline 9 & 16,321 & $20,971.2$ & $21,362.7$ & $20,971.0$ & 0 & -1.83 \\
\hline 10 & 17,998 & $22,645.8$ & $22,647.5$ & $22,562.8$ & -0.37 & -0.37 \\
\hline 11 & 11,080 & $12,955.6$ & $12,955.6$ & $12,955.3$ & 0 & 0 \\
\hline 12 & 24,721 & $26,831.3$ & $26,831.3$ & $26,831.1$ & 0 & 0 \\
\hline 13 & 20,782 & $23,127.8$ & $23,127.8$ & $23,128.5$ & 0 & 0 \\
\hline 14 & 22,264 & $25,035.8$ & $25,035.8$ & $25,036.0$ & 0 & 0 \\
\hline 15 & 12,401 & $14,118.1$ & $14,118.1$ & $14,117.9$ & 0 & 0 \\
\hline 16 & 15,122 & $17,540.2$ & $17,400.1$ & $17,400.1$ & -0.80 & 0 \\
\hline 17 & 20,468 & $23,007.5$ & $23,007.5$ & $22,996.2$ & -0.05 & -0.05 \\
\hline 18 & 11,075 & $12,973.8$ & $12,973.8$ & $12,973.8$ & 0 & 0 \\
\hline 19 & 13,276 & $16,502.9$ & $16,502.9$ & $16,349.2$ & -0.93 & -0.93 \\
\hline
\end{tabular}


Table 4 continued

\begin{tabular}{|c|c|c|c|c|c|c|}
\hline \multirow[t]{2}{*}{ SET1 } & \multirow[t]{2}{*}{ XLP } & \multicolumn{3}{|c|}{ Solution values } & \multicolumn{2}{|c|}{ Deviation (\%) } \\
\hline & & Aheur & LugNP & RFFO & Aheur & LugNP \\
\hline 20 & 14,101 & $17,158.6$ & $17,158.6$ & $17,158.7$ & 0 & 0 \\
\hline 21 & 10,159 & $12,421.2$ & $12,421.2$ & $12,421.1$ & 0 & 0 \\
\hline 22 & 38,040 & $40,158.3$ & $40,188.7$ & $40,158.4$ & 0 & -0.08 \\
\hline 23 & 29,331 & $30,605.7$ & $30,605.7$ & $30,605.5$ & 0 & 0 \\
\hline 24 & 28,858 & $32,190.4$ & $32,145.5$ & $32,007.2$ & -0.57 & -0.43 \\
\hline 25 & 51,371 & $52,989.2$ & $52,959.9$ & $52,960.3$ & -0.05 & 0 \\
\hline 26 & 39,379 & $41,221.5$ & $41,221.5$ & $41,221.0$ & 0 & 0 \\
\hline 27 & 40,838 & $43,319.7$ & $43,319.7$ & $43,289.6$ & -0.07 & -0.07 \\
\hline 28 & 39,846 & $40,993.5$ & $41,019.8$ & $40,993.5$ & 0 & -0.06 \\
\hline 29 & 23,155 & $25,492.6$ & $25,322.3$ & $25,322.0$ & -0.67 & 0 \\
\hline 30 & 68,989 & $70,863.7$ & $70,863.7$ & $70,863.7$ & 0 & 0 \\
\hline
\end{tabular}

Table 5 Comparison for SET2 instances (time limit = $150 \mathrm{~s}$ )

\begin{tabular}{|c|c|c|c|c|c|c|}
\hline \multirow[t]{2}{*}{ SET2 } & \multirow[t]{2}{*}{ XLP } & \multicolumn{3}{|c|}{ Solution values } & \multicolumn{2}{|c|}{ Deviation (\%) } \\
\hline & & Aheur & LugNP & RFFO & Aheur & LugNP \\
\hline 1 & 46,116 & $52,050.7$ & $52,050.7$ & $52,339.4$ & 0.55 & 0.55 \\
\hline 2 & 47,780 & $53,863.4$ & $53,713.4$ & $53,713.0$ & -0.28 & 0 \\
\hline 3 & 40,551 & $46,894.5$ & $47,053.2$ & $46,893.3$ & 0 & -0.34 \\
\hline 4 & 36,347 & $43,009.8$ & $42,977.1$ & $43,063.1$ & 0.12 & 0.20 \\
\hline 5 & 45,395 & $51,757.6$ & $51,757.6$ & $51,768.8$ & 0.02 & 0.02 \\
\hline 6 & 45,902 & $51,858.1$ & $51,858.1$ & $51,858.4$ & 0 & 0 \\
\hline 7 & 52,825 & $58,153.8$ & $58,153.8$ & $58,425.2$ & 0.47 & 0.47 \\
\hline 8 & 48,033 & $54,396.2$ & $54,449.6$ & $54,182.9$ & -0.39 & -0.49 \\
\hline 9 & 37,553 & $43,737.8$ & $43,737.8$ & $43,690.0$ & -0.11 & -0.11 \\
\hline 10 & 38,751 & $45,278.8$ & $45,278.8$ & $45,305.8$ & 0.06 & 0.06 \\
\hline 11 & 65,210 & $68,488.8$ & $68,646.4$ & $68,487.8$ & 0 & -0.23 \\
\hline 12 & 62,792 & $66,561.9$ & $66,474.5$ & $66,475.4$ & -0.13 & 0 \\
\hline 13 & 34,778 & $39,120.3$ & $39,082.7$ & $38,852.7$ & -0.68 & -0.59 \\
\hline 14 & 62,907 & $66,373.7$ & $66,383.2$ & $66,325.1$ & -0.07 & -0.09 \\
\hline 15 & 59,079 & $61,574.1$ & $61,574.1$ & $61,574.0$ & 0 & 0 \\
\hline 16 & 75,682 & $79,364.8$ & $79,385.0$ & $79,363.9$ & 0 & -0.03 \\
\hline 17 & 36,809 & $41,298.6$ & $41,282.4$ & $41,192.6$ & -0.26 & -0.22 \\
\hline 18 & 77,873 & $81,561.8$ & $81,562.9$ & $81,562.5$ & 0 & 0 \\
\hline 19 & 54,981 & $58,426.1$ & $58,426.1$ & $58,425.4$ & 0 & 0 \\
\hline 20 & 119,568 & $122,827.6$ & $122,827.6$ & $122,829.0$ & 0 & 0 \\
\hline
\end{tabular}


Table 5 continued

\begin{tabular}{|c|c|c|c|c|c|c|}
\hline \multirow[t]{2}{*}{ SET2 } & \multirow[t]{2}{*}{ XLP } & \multicolumn{3}{|c|}{ Solution values } & \multicolumn{2}{|c|}{ Deviation (\%) } \\
\hline & & Aheur & LugNP & RFFO & Aheur & LugNP \\
\hline 21 & 22,281 & $24,013.2$ & $24,014.2$ & $24,013.3$ & 0 & 0 \\
\hline 22 & 51,279 & $52,887.1$ & $52,887.1$ & $52,886.8$ & 0 & 0 \\
\hline 23 & 29,793 & $32,618.2$ & $32,708.8$ & $33,713.9$ & 3.36 & 3.07 \\
\hline 24 & 65,891 & $68,640.6$ & $68,575.1$ & $68,574.8$ & -0.10 & 0 \\
\hline 25 & 75,627 & $78,064.3$ & $78,088.2$ & $78,064.2$ & 0 & -0.03 \\
\hline 26 & 60,952 & $63,275.2$ & $63,285.6$ & $63,273.2$ & 0 & -0.02 \\
\hline 27 & 53,016 & $54,794.1$ & $54,794.1$ & $54,793.9$ & 0 & 0 \\
\hline 28 & 44,545 & $46,607.9$ & $46,607.9$ & $46,607.6$ & 0 & 0 \\
\hline 29 & 93,631 & $96,278.0$ & $96,157.4$ & $96,152.0$ & -0.13 & 0 \\
\hline 30 & 68,324 & $71,408.0$ & $71,408.0$ & $71,408.7$ & 0 & 0 \\
\hline
\end{tabular}

Table 6 Comparison for SET3 instances (time limit $=300 \mathrm{~s})$

\begin{tabular}{|c|c|c|c|c|c|c|}
\hline \multirow[t]{2}{*}{ SET3 } & \multirow[t]{2}{*}{ XLP } & \multicolumn{3}{|c|}{ Solution values } & \multicolumn{2}{|c|}{ Deviation (\%) } \\
\hline & & Aheur & LugNP & RFFO & Aheur & LugNP \\
\hline 1 & 65,668 & $188,294.0$ & $189,400.6$ & $179,554.0$ & -4.64 & -5.20 \\
\hline 2 & 82,342 & $216,700.4$ & $217,283.4$ & $216,401.0$ & -0.14 & -0.41 \\
\hline 3 & 74,209 & $216,517.4$ & $207,362.6$ & $198,215.0$ & -8.45 & -4.41 \\
\hline 4 & 78,282 & $214,175.7$ & $220,062.4$ & $203,208.0$ & -5.12 & -7.66 \\
\hline 5 & 76,607 & $220,928.0$ & $220,686.4$ & $201,723.0$ & -8.69 & -8.59 \\
\hline 6 & 79,093 & $213,987.2$ & $210,339.0$ & $203,253.0$ & -5.02 & -3.37 \\
\hline 7 & 72,979 & $206,793.3$ & $208,245.8$ & $193,804.0$ & -6.28 & -6.93 \\
\hline 8 & 88,610 & $231,333.9$ & $224,404.5$ & $226,042.0$ & -2.29 & 0.73 \\
\hline 9 & 64,180 & $198,594.1$ & $183,327.9$ & $178,576.0$ & -10.08 & -2.59 \\
\hline 10 & 66,878 & $201,771.0$ & $192,069.0$ & $188,790.0$ & -6.43 & -1.71 \\
\hline 11 & 42,946 & $132,466.6$ & $130,055.9$ & $132,231.0$ & -0.18 & 1.67 \\
\hline 12 & 86,047 & $213,445.5$ & $211,726.2$ & $195,981.0$ & -8.18 & -7.44 \\
\hline 13 & 74,643 & $199,471.6$ & $197,240.0$ & $195,772.0$ & -1.85 & -0.74 \\
\hline 14 & 85,209 & $198,005.1$ & $200,193.9$ & $200,257.0$ & 1.14 & 0.03 \\
\hline 15 & 40,715 & $135,491.1$ & $125,875.5$ & $127,045.0$ & -6.23 & 0.93 \\
\hline 16 & 46,548 & $144,580.2$ & $149,411.0$ & $135,689.0$ & -6.15 & -9.18 \\
\hline 17 & 71,555 & $200,971.1$ & $199,875.3$ & $184,830.0$ & -8.03 & -7.53 \\
\hline 18 & 39,533 & $98,901.8$ & $97,031.1$ & $98,106.0$ & -0.80 & 1.11 \\
\hline 19 & 47,495 & $149,973.9$ & $151,618.8$ & $138,420.0$ & -7.70 & -8.71 \\
\hline 20 & 58,189 & $170,524.4$ & $163,785.9$ & $163,740.0$ & -3.98 & -0.03 \\
\hline
\end{tabular}


Table 6 continued

\begin{tabular}{|c|c|c|c|c|c|c|}
\hline \multirow[t]{2}{*}{ SET3 } & \multirow[t]{2}{*}{ XLP } & \multicolumn{3}{|c|}{ Solution values } & \multicolumn{2}{|c|}{ Deviation (\%) } \\
\hline & & Aheur & LugNP & RFFO & Aheur & LugNP \\
\hline 21 & 44,182 & $141,578.2$ & $134,625.9$ & $124,919.0$ & -11.77 & -7.21 \\
\hline 22 & 130,235 & $256,283.6$ & $245,549.4$ & $246,270.0$ & -3.91 & 0.29 \\
\hline 23 & 96,810 & $229,468.8$ & $215,893.6$ & $209,798.0$ & -8.57 & -2.82 \\
\hline 24 & 105,300 & $272,965.6$ & $245,491.9$ & $241,071.0$ & -11.68 & -1.80 \\
\hline 25 & 203,044 & $329,382.0$ & $333,236.6$ & $324,800.0$ & -1.39 & -2.53 \\
\hline 26 & 145,184 & $286,229.0$ & $289,459.6$ & $280,060.0$ & -2.16 & -3.25 \\
\hline 27 & 145,420 & $294,614.0$ & $297,025.5$ & $286,754.0$ & -2.67 & -3.46 \\
\hline 28 & 145,227 & $225,567.2$ & $224,734.0$ & $227,483.0$ & 0.85 & 1.22 \\
\hline 29 & 79,813 & $189,879.7$ & $185,569.7$ & $191,242.0$ & 0.72 & 3.06 \\
\hline 30 & 274,018 & $415,185.0$ & $407,150.8$ & $399,907.0$ & -3.68 & -1.78 \\
\hline
\end{tabular}

Table 7 Comparison for SET4 instances (time limit $=300 \mathrm{~s}$ )

\begin{tabular}{|c|c|c|c|c|c|c|}
\hline \multirow[t]{2}{*}{ SET4 } & \multirow[t]{2}{*}{ XLP } & \multicolumn{3}{|c|}{ Solution values } & \multicolumn{2}{|c|}{ Deviation (\%) } \\
\hline & & Aheur & LugNP & RFFO & Aheur & LugNP \\
\hline 1 & 16,353 & $57,483.0$ & $53,168.4$ & $53,062.3$ & -7.69 & -0.20 \\
\hline 2 & 31,541 & $80,772.8$ & $77,346.7$ & $73,884.2$ & -8.53 & -4.48 \\
\hline 3 & 24,864 & $68,176.9$ & $67,097.7$ & $66,030.7$ & -3.15 & -1.59 \\
\hline 4 & 27,786 & $72,989.4$ & $68,995.1$ & $68,662.6$ & -5.93 & -0.48 \\
\hline 5 & 25,450 & $67,329.1$ & $66,993.7$ & $66,328.8$ & -1.49 & -0.99 \\
\hline 6 & 30,632 & $75,042.4$ & $74,601.8$ & $70,698.3$ & -5.79 & -5.23 \\
\hline 7 & 22,650 & $62,993.3$ & $64,132.5$ & $62,974.3$ & -0.03 & -1.81 \\
\hline 8 & 40,532 & $81,200.6$ & $84,586.1$ & $80,914.5$ & -0.35 & -4.34 \\
\hline 9 & 13,490 & $55,901.5$ & $52,041.0$ & $51,457.5$ & -7.95 & -1.12 \\
\hline 10 & 15,542 & $55,602.2$ & $57,297.3$ & $55,341.7$ & -0.47 & -3.41 \\
\hline 11 & 12,802 & $28,415.7$ & $28,323.5$ & $28,207.1$ & -0.73 & -0.41 \\
\hline 12 & 43,341 & $73,653.4$ & $72,084.8$ & $71,886.9$ & -2.40 & -0.27 \\
\hline 13 & 28,152 & $52,525.0$ & $55,251.9$ & $52,518.4$ & -0.01 & -4.95 \\
\hline 14 & 56,174 & $79,086.4$ & $80,501.7$ & $78,903.9$ & -0.23 & -1.98 \\
\hline 15 & 14,628 & $25,927.5$ & $25,286.3$ & $24,568.9$ & -5.24 & -2.84 \\
\hline 16 & 17,171 & $35,048.5$ & $35,138.7$ & $34,569.2$ & -1.37 & -1.62 \\
\hline 17 & 29,001 & $51,396.2$ & $51,671.9$ & $51,266.5$ & -0.25 & -0.78 \\
\hline 18 & 19,184 & $26,101.5$ & $26,282.3$ & $26,037.4$ & -0.25 & -0.93 \\
\hline 19 & 10,724 & $31,585.8$ & $33,006.4$ & $31,139.0$ & -1.41 & -5.66 \\
\hline 20 & 18,718 & $38,796.1$ & $38,781.4$ & $37,179.1$ & -4.17 & -4.13 \\
\hline
\end{tabular}


Table 7 continued

\begin{tabular}{|c|c|c|c|c|c|c|}
\hline \multirow[t]{2}{*}{ SET3 } & \multirow[t]{2}{*}{ XLP } & \multicolumn{3}{|c|}{ Solution values } & \multicolumn{2}{|c|}{ Deviation $(\%)$} \\
\hline & & Aheur & LugNP & RFFO & Aheur & LugNP \\
\hline 21 & 15,812 & $25,727.0$ & $25,840.8$ & $25,713.0$ & -0.05 & -0.49 \\
\hline 22 & 91,715 & $120,008.2$ & $119,481.0$ & $118,749.0$ & -1.05 & -0.61 \\
\hline 23 & 55,058 & $74,180.4$ & $73,297.4$ & $73,296.6$ & -1.19 & 0 \\
\hline 24 & 58,919 & $82,349.4$ & $82,260.2$ & $80,733.2$ & -1.96 & -1.86 \\
\hline 25 & 171,987 & $196,626.7$ & $196,025.1$ & $196,023.0$ & -0.31 & 0 \\
\hline 26 & 110,570 & $137,224.6$ & $134,856.0$ & $134,854.0$ & -1.73 & 0 \\
\hline 27 & 101,114 & $135,936.6$ & $132,463.3$ & $132,451.0$ & -2.56 & 0 \\
\hline 28 & 112,892 & $126,553.7$ & $126,157.1$ & $125,872.0$ & -0.54 & -0.23 \\
\hline 29 & 51,149 & $66,131.1$ & $66,217.4$ & $66,131.4$ & 0 & -0.13 \\
\hline 30 & 241,678 & $262,380.7$ & $263,042.1$ & $262,378.0$ & 0 & -0.25 \\
\hline
\end{tabular}

\section{References}

Absi, N., Detienne, B., Dauzère-Pérès, S.: Heuristics for the multi-item capacitated lot-sizing problem with lost sales. Comput. Oper. Res. 40(1), 264-272 (2013)

Akartunalı, K., Miller, A.J.: A heuristic approach for big bucket multi-level production planning problems. Eur. J. Oper. Res. 193(2), 396-411 (2009)

Akartunalı, K., Miller, A.J.: A computational analysis of lower bounds for big bucket production planning problems. Comput. Optim. Appl. 53(3), 729-753 (2012)

Almada-Lobo, B., Klabjan, D., Carravilla, M.A., Oliveira, J.F.: Multiple machine continuous setup lotsizing with sequence-dependent setups. Comput. Optim. Appl. 47(3), 529-552 (2010)

Almeder, C.: A hybrid optimization approach for multi-level capacitated lot-sizing problems. Eur. J. Oper. Res. 200, 599-606 (2010)

Baki, M.F., Chaouch, B.A., Abdul-Kader, W.: A heuristic solution procedure for the dynamic lot sizing problem with remanufacturing and product recovery. Comput. Oper. Res. 43, 225-236 (2014)

Ball, M.O.: Heuristics based on mathematical programming. Surv. Oper. Res. Manage. Sci. 16(1), 21-38 (2011)

Barany, I., van Roy, T.J., Wolsey, L.A.: Uncapacitated lot sizing: the convex hull of solutions. Math. Program Study 22, 32-43 (1984)

Belvaux, G., Wolsey, L.A.: bc-prod: a specialized branch-and-cut system for lot-sizing problems. Manage. Sci. 46(5), 724-738 (2000)

Billington, P.J., McClain, J.O., Thomas, L.J.: Heuristics for multilevel lot-sizing with a bottleneck. Manage. Sci. 32, 989-1006 (1986)

Degraeve, Z., Jans, R.: A new Dantzig-Wolfe reformulation and branch-and-price algorithm for the capacitated lot-sizing problem with setup times. Oper. Res. 55(5), 909-920 (2007)

Eppen, G.D., Martin, R.K.: Solving multi-item capacitated lot-sizing problems using variable redefinition. Oper. Res. 35(6), 832-848 (1987)

Federgruen, A., Meissner, J., Tzur, M.: Progressive interval heuristics for multi-item capacitated lot sizing problem. Oper. Res. 55(3), 490-502 (2007)

Florian, M., Lenstra, J.K., Rinnooy Kan, H.G.: Deterministic production planning: algorithms and complexity. Manage. Sci. 26(7), 669-679 (1980)

Helber, S., Sahling, F.: A fix-and-optimize approach for the multi-level capacitated lot sizing problem. Int. J. Prod. Econ. 123, 247-256 (2010)

Kébé, S., Sbihi, N., Penz, B.: A lagrangean heuristic for a two-echelon storage capacitated lot-sizing problem. J. Intell. Manuf. 23(6), 2477-2483 (2012) 
Krarup, J., Bilde, O.: Plant location, set covering and economic lotsizes: an $\mathrm{O}(m n)$ algorithm for structured problems. Optimierung bel Graphentheoretischen und Ganzzahligen Probleme, pp. 155-180. Birkhauser (1997)

Küçükyavuz, S., Pochet, Y.: Uncapacitated lot-sizing with backlogging: The convex hull. Math. Program. 118(1), 151-175 (2009)

Miller, A.J., Nemhauser, G.L., Savelsbergh, M.W.P.: On the polyhedral structure of a multi-item production planning model with setup times. Math. Program. 94(2-3), 375-405 (2003)

MIPLIB. A library of pure and mixed integer problems (2010). http://miplib.zib.de/. Accessed on 29 Dec 2014

Multi-LSB. Multi-item lot-sizing problems with backlogging: a library of test instances (2014). http://dx. doi.org/10.15129/252b7827-b62b-4af4-8869-64b12b1c69a1. Accessed 29 Dec 2014

Pochet, Y., Wolsey, L.A.: Production Planning by Mixed Integer Programming. Springer, Berlin (2006)

Ramezanian, R., Saidi-Mehrabad, M.: Hybrid simulated annealing and mip-based heuristics for stochastic lot-sizing and scheduling problem in capacitated multi-stage production system. Appl Math Modell 37(7), 5134-5147 (2013)

Rardin, R.L., Wolsey, L.A.: Valid inequalities and projecting the multicommodity extended formulation for uncapacitated fixed charge network flow problems. Eur. J. Oper. Res. 71(1), 95-109 (1993)

Seeanner, F., Almada-Lobo, B., Meyr, H.: Combining the principles of variable neighborhood decomposition search and the fix \& optimize heuristic to solve multi-level lot-sizing and scheduling problems. Comput. Oper. Res. 40(1), 303-317 (2013)

Stadtler, H.: Multilevel lot sizing with setup times and multiple constrained resources: internally rolling schedules with lot-sizing windows. Oper. Res. 51, 487-502 (2003)

Toledo, C.F.M., da Silva Arantes, M., de Oliveira, R.R.R., Almada-Lobo, B.: Glass container production scheduling through hybrid multi-population based evolutionary algorithm. Appl. Soft Comput. 13(3), 1352-1364 (2013)

Toledo, C.F.M., de Oliveira, R.R.R., França, P.M.: A hybrid multi-population genetic algorithm applied to solve the multi-level capacitated lot sizing problem with backlogging. Comput. Oper. Res. 40(4), 910-919 (2013)

Van Vyve, M., Pochet, Y.: A general heuristic for production planning problems. INFORMS J. Comput. 16(3), 316-327 (2004)

Wu, T., Shi, L., Geunes, J., Akartunal, K.: An optimization framework for solving capacitated multi-level lot-sizing problems with backlogging. Eur. J. Oper. Res. 214(2), 428-441 (2011) 